\title{
ON THE CAUCHY PROBLEM OF A DEGENERATE PARABOLIC-HYPERBOLIC PDE WITH LÉVY NOISE
}

\author{
IMRAN H. BISWAS, ANANTA K. MAJEE, AND GUY VALLET
}

\begin{abstract}
In this article we deal with stochastic perturbation of degenerate parabolic partial differential equations (PDEs). The particular emphasise is on analysing the effect of multiplicative Lévy noise to such problems and establishing wellposedness by developing a suitable weak entropy solution framework. The proof of existence is based on the vanishing viscosity technique. The uniqueness is settled by interpreting Kruzkov's doubling technique in the presence noise.
\end{abstract}

\section{Contents}

1. Introduction

1.1. Studies on degenerate parabolic-hyperbolic equations with Brownian noise

1.2. Relevant studies on problems with Lévy noise

1.3. Goal of the study and outline of the paper

2. Technical framework and statements of the main results

2.1. Entropy inequalities

3. Existence of weak solution for viscous problem

3.1. Existence of weak solution to viscous problem

3.2. A priori bounds for viscous solutions

4. Existence of entropy solution

4.1. Uniqueness of Young measure valued limit process

4.2. Existence of entropy solution

5. Uniqueness of entropy solution

Appendix A. Weak Itô-Lévy formula

References

\section{InTRODUCTION}

Let $\left(\Omega, P, \mathcal{F},\left\{\mathcal{F}_{t}\right\}_{t \geq 0}\right)$ be a filtered probability space satisfying the usual hypothesis i.e. $\left\{\mathcal{F}_{t}\right\}_{t \geq 0}$ is a right-continuous filtration such that $\mathcal{F}_{0}$ contains all the $P$-null subsets of $(\Omega, \mathcal{F})$. In addition, let $(E, \mathcal{E}, m)$ be a $\sigma$-finite measure space and $N(d t, d z)$ be a Poisson random measure on $(E, \mathcal{E})$ with intensity measure $m(d z)$ with respect to the same stochastic basis. The existence and construction of such general notion of Poisson random measure with a given intensity measure are detailed in 22]. We are interested in the Cauchy problem for a nonlinear degenerate parabolic stochastic PDE of the following type

$$
d u(t, x)-\Delta \phi(u(t, x)) d t-\operatorname{div}_{x} f(u(t, x)) d t=\int_{E} \eta(x, u(t, x) ; z) \tilde{N}(d z, d t), \quad(t, x) \in \Pi_{T},
$$

with the initial condition

$$
u(0, x)=u_{0}(x), \quad x \in \mathbb{R}^{d},
$$

2000 Mathematics Subject Classification. 45K05, 46S50, 49L20, 49L25, 91A23, 93E20.

Key words and phrases. Stochastic PDEs, Lévy noise, degenerate parabolic equations, entropy solutions, Young measures.

The authors are profoundly thankful for the generous support from IFCAM which allowed them to travel between India \& France and made this collaboration possible. The third author would like to also acknowledge the support of ISIFoR. . 
where $\Pi_{T}=[0, T) \times \mathbb{R}^{d}$ with $T>0$ fixed, $u(t, x)$ is the unknown random scalar valued function, $F: \mathbb{R} \rightarrow \mathbb{R}^{d}$ is given flux function, and $\tilde{N}(d z, d t)=N(d z, d t)-m(d z) d t$, the compensated Poisson random measure. Furthermore, $(x, u, z) \mapsto \eta(x, u ; z)$ is a real valued function defined on the domain $\mathbb{R}^{d} \times \mathbb{R} \times E$ and $\phi: \mathbb{R} \rightarrow \mathbb{R}$ is a given non-decreasing Lipschitz continuous function. The stochastic integral in the RHS of (1.1) is defined in the Lévy-Itô sense.

Remark 1.1. Since $\phi$ is a real valued non-decreasing, Lipschitz continuous function, the set $A=\left\{r \in \mathbb{R}: \phi^{\prime}(r)=0\right\}$ is not empty in general and hence the problem is called degenerate. Even more, $A$ is not negligible either and the problem is strongly degenerate in the sense of [10].

Remark 1.2. The analysis of this paper remains valid if the noise on the RHS of (1.1) is of jumpdiffusion type. In other words, the same analysis holds if we add a $\sigma(x, u) d W_{t}$ term in the RHS of (1.1) where $W_{t}$ is a cylindrical Brownian motion. Moreover, we will carry out our analysis under the structural assumption $E=\mathcal{O} \times \mathbb{R}^{*}$ where $\mathcal{O}$ is a subset of the Euclidean space. The measure $m$ on $E$ is defined as $\lambda \times \mu$ where $\lambda$ is a Radon measure on $\mathcal{O}$ and $\mu$ is a so called Lévy measure on $\mathbb{R}^{*}$. In such a case, the noise of the RHS would be called an impulsive white noise with jump position intensity $\lambda$ and jump size intensity $\mu$. We refer to 22 for more on Lévy sheet and related impulsive white noise.

The equation (1.1) becomes a multidimensional deterministic degenerate parabolic-hyperbolic equation if $\eta=0$. It is well-documented in the literature that the solution has to be interpreted in the weak sense and one needs an entropy formulation to prove wellposedness. We refer to [1, 10, 12, 13, 5, 25] and references therein for more on entropy solution theory for deterministic degenerate parabolic-hyperbolic equations.

1.1. Studies on degenerate parabolic-hyperbolic equations with Brownian noise. The study of stochastic degenerate parabolic-hyperbolic equations has so far been limited to mainly equations with Brownian noise. In particular, hyperbolic conservation laws with Brownian noise are the examples of such problems that have attracted the attention of many. The first documented development in this direction is [19], where the authors established existence of path-wise weak solution (possibly non-unique) of one dimensional balance laws via splitting method. In a separate development, Khanin et al. 20, published their celebrated work that described some statistical properties of Burgers equations with noise. J. U. Kim [21] extended Kruzkov's entropy formulation and established the wellposedness for one dimensional balance laws that are driven by additive Brownian noise. Multidimensional case was studied by Vallet and Wittbold 223, and they established wellposedness of entropy solution with the theory of Young-measures but in a bounded domain.

This approach is not applicable for multiplicative noise case. This was studied by many authors (4, 11, 15, 18]). In [18, Feng and Nualart came up with a way to recover the necessary information in the form of strong entropy condition from the parabolic regularisation and established the uniqueness of strong entropy solution in $L^{p}$-framework for several space dimensions but the existence was for one space dimension. We also add here that Feng and Nualart [18] uses an entropy formulation which is strong in time but weak in space, which in our view may give rise to problems where the solutions are not shown to have continuous sample paths. We refer to 6], where a few technical questions are raised on the strong in time formulation and remedial measures have been proposed. In [15, the authors obtain the existence via kinetic formulation and [1] uses BV solution framework. In a recent paper, Vallet et al. 4, established the wellposedness via the Young's measure approach. The wellposedness result of the multidimensional degenerate parabolic-hyperbolic stochastic problem has been studied by Vovelle, Hofmanova and Debussche 14, and Vallet et al. 3. In [14, they adapt the notion of kinetic formulation and develop a wellposedness theory. In [3, the authors revisited [1, 10, 12] and established the wellposedness of the entropy solution via Young's measure theory.

1.2. Relevant studies on problems with Lévy noise. Over the last decade there has been many contributions on the larger area of stochastic partial differential equations that are driven by Lévy noise. An worthy reference on this subject is 22. However, very little is available on the specific problem of degenerate parabolic problems with Lévy noise such as (1.1). This article marks 
an important step in our quest to develop a comprehensive theory of stochastic degenerate parabolic equations that are driven by jump-diffusions. The relevant results in this context are made available recently and they are on conservation laws that are perturbed by Lévy noise. In recent articles [7. 8], Biswas et al. established existence, uniqueness of entropy solution for multidimensional conservation laws with Poisson noise via Young measure approach. In [8], the authors developed a continuous dependence theory on nonlinearities within $B V$ solution setting.

Stochastic degenerate parabolic-hyperbolic equations are one of the most important classes of nonlinear stochastic PDEs. Nonlinearity and degeneracy are two main features of these equations and yield several striking phenomena. Therefore, it requires new mathematical ideas, approaches, and theories. It is well-known that due to presence of nonlinear flux term, solutions to (1.1) are not smooth even for smooth initial data $u_{0}(x)$. Therefore the solutions must be interpreted in the weak sense. Before introducing the concept of weak solutions, we first recall the notion of predictable $\sigma$-field. By a predictable $\sigma$-field on $[0, T] \times \Omega$, denoted by $\mathcal{P}_{T}$, we mean that the $\sigma$-field generated by the sets of the form: $\{0\} \times A$ and $(s, t] \times B$ for any $A \in \mathcal{F}_{0} ; B \in \mathcal{F}_{s}, 0<s, t \leq T$. The notion of stochastic weak solution is defined as follows.

Definition 1.1 (Stochastic weak solution). An $L^{2}\left(\mathbb{R}^{d}\right)$-valued $\left\{\mathcal{F}_{t}: t \geq 0\right\}$-predictable stochastic process $u(t)=u(t, x)$ is said to be a weak solution to our problem (1.1) provided

1) $u \in L^{2}\left(\Omega \times \Pi_{T}\right)$ and $\phi(u) \in L^{2}\left((0, T) \times \Omega ; H^{1}\left(\mathbb{R}^{d}\right)\right)$.

(2) $\frac{\partial}{\partial t}\left[u-\int_{0}^{t} \int_{E} \eta(x, u(s, \cdot) ; z) \tilde{N}(d z, d s)\right] \in L^{2}\left((0, T) \times \Omega ; H^{-1}\left(\mathbb{R}^{d}\right)\right)$ in the sense of distribution.

(3) For almost every $t \in[0, T]$ and $P-$ a.s, the following variational formulation holds:

$$
\begin{aligned}
0=\left\langle\frac{\partial}{\partial t}[u\right. & \left.\left.-\int_{0}^{t} \int_{E} \eta(x, u(s, \cdot) ; z) \tilde{N}(d z, d s)\right], v\right\rangle_{H^{-1}\left(\mathbb{R}^{d}\right), H^{1}\left(\mathbb{R}^{d}\right)} \\
& +\int_{\mathbb{R}^{d}}\{\nabla \phi(u(t, x))+f(u(t, x))\} \cdot \nabla v d x
\end{aligned}
$$

for any $v \in H^{1}\left(\mathbb{R}^{d}\right)$.

However, it is well-known that weak solutions may be discontinuous and they are not uniquely determined by their initial data. Consequently, an admissibility criterion for so called entropy solution (see Section 2 for the definition of entropy solution) must be imposed to single out the physically correct solution.

1.3. Goal of the study and outline of the paper. The case of a strongly degenerate stochastic problem driven by Brownian noise is studied by Bauzet et al. 33. In this article, drawing primary motivation from [3, 7, 10, we propose to establish the wellposedness of the entropy solution to degenerate Cauchy problem (1.1) by using vanishing viscosity method along with few a priori bounds.

The rest of the paper is organized as follows. We state the assumptions, details of the technical framework and state the main results in Section 2. Section 3 is devoted to prove the existence of weak solution for viscous problem via implicit time discretization scheme and to derive some a priori estimates for viscous solution. In section 4, we first establish uniqueness of the limit of viscous solutions as viscous parameter goes to zero via Young measure theory and then we establish existence of entropy solution. The uniqueness of the entropy solution is presented in the final section.

\section{TECHNiCAL FRAMEWORK AND STATEMENTS OF THE MAIN RESUlts}

Here and in the sequel, we denote by $N_{\omega}^{2}\left(0, T, L^{2}\left(\mathbb{R}^{d}\right)\right)$ the space of predictable $L^{2}\left(\mathbb{R}^{d}\right)$-valued processes $u$ such that $\mathbb{E}\left[\int_{\Pi_{T}}|u|^{2} d t d x\right]<+\infty$. Moreover, we use the letter $C$ to denote various generic constants. There are situations where constants may change from line to line, but the notation is kept unchanged so long as it does not impact the primary implication. We denote $c_{\phi}$ and $c_{f}$ the Lipschitz constants of $\phi$, and $f$ respectively. Also, we use $\langle$,$\rangle to denote the pairing$ between $H^{1}\left(\mathbb{R}^{d}\right)$ and $H^{-1}\left(\mathbb{R}^{d}\right)$. 
2.1. Entropy inequalities. We begin this subsection with a formal derivation of entropy inequalities à la Kruzkov. Remember that we need to replace the traditional chain rule for deterministic calculus by Itô-Lévy chain rule.

Definition 2.1 (Entropy flux triple). A triplet $(\beta, \zeta, \nu)$ is called an entropy flux triple if $\beta \in C^{2}(\mathbb{R})$, Lipschitz and $\beta \geq 0, \zeta=\left(\zeta_{1}, \zeta_{2}, \ldots \zeta_{d}\right): \mathbb{R} \mapsto \mathbb{R}^{d}$ is a vector valued function, and $\nu: \mathbb{R} \mapsto \mathbb{R}$ is a scalar valued function such that

$$
\zeta^{\prime}(r)=\beta^{\prime}(r) f^{\prime}(r) \quad \text { and } \quad \nu^{\prime}(r)=\beta^{\prime}(r) \phi^{\prime}(r) .
$$

An entropy flux triple $(\beta, \zeta, \nu)$ is called convex if $\beta^{\prime \prime}(s) \geq 0$.

For a small positive number $\varepsilon>0$, assume that the parabolic perturbation

$$
\begin{aligned}
d u(t, x)-\Delta \phi(u(t, x)) d t=\operatorname{div}_{x} f(u(t, x)) d t+ & \int_{E} \eta(x, u(t, x) ; z) \tilde{N}(d z, d t) \\
& +\varepsilon \Delta u(t, x) d t, \quad(t, x) \in \Pi_{T}
\end{aligned}
$$

of (1.1) has a unique weak solution $u_{\varepsilon}(t, x)$. Note that this weak solution $u_{\varepsilon} \in L^{2}((0, T) \times$ $\left.\Omega ; H^{1}\left(\mathbb{R}^{d}\right)\right)$. Moreover, for the time being, we assume that it satisfies the initial condition in the sense of (A.2). This enables one to derive a weak version of Itô -Lévy formula for the solutions to (1.1), as detailed in the Theorem A.1 in the Appendix.

Let $(\beta, \zeta, \nu)$ be an entropy flux triple. Given a nonnegative test function $\psi \in C_{c}^{1,2}\left([0, \infty) \times \mathbb{R}^{d}\right)$, we apply generalised version of the Itô-Lévy formula to have, for almost every $T>0$,

$$
\begin{aligned}
& \int_{\mathbb{R}^{d}} \beta\left(u_{\varepsilon}(T, x)\right) \psi(T, x) d x-\int_{\mathbb{R}^{d}} \beta\left(u_{\varepsilon}(0, x)\right) \psi(0, x) d x \\
= & \int_{\Pi_{T}} \beta\left(u_{\varepsilon}(t, x)\right) \partial_{t} \psi(t, x) d x d t-\int_{\Pi_{T}} \nabla \psi(t, x) \cdot \zeta\left(u_{\varepsilon}(t, x)\right) d x d t \\
+ & \int_{\Pi_{T}} \int_{E} \int_{0}^{1} \eta\left(x, u_{\varepsilon}(t, x) ; z\right) \beta^{\prime}\left(u_{\varepsilon}(t, x)+\theta \eta\left(x, u_{\varepsilon}(t, x) ; z\right)\right) \psi(t, x) d \theta \tilde{N}(d z, d t) d x \\
+ & \int_{\Pi_{T}} \int_{E} \int_{0}^{1}(1-\theta) \eta^{2}\left(x, u_{\varepsilon}(t, x) ; z\right) \beta^{\prime \prime}\left(u_{\varepsilon}(t, x)+\theta \eta\left(x, u_{\varepsilon}(t, x) ; z\right)\right) \psi(t, x) d \theta m(d z) d x d t \\
- & \int_{\Pi_{T}}\left(\varepsilon \nabla_{x} \psi(t, x) \cdot \nabla_{x} \beta\left(u_{\varepsilon}(t, x)\right)+\varepsilon \beta^{\prime \prime}\left(u_{\varepsilon}(t, x)\right)\left|\nabla_{x} u_{\varepsilon}(t, x)\right|^{2} \psi(t, x)\right) d x d t \\
- & \int_{\Pi_{T}} \phi^{\prime}\left(u_{\varepsilon}(t, x)\right) \beta^{\prime \prime}\left(u_{\varepsilon}(t, x)\right)\left|\nabla u_{\varepsilon}(t, x)\right|^{2} \psi(t, x) d x d t+\int_{\Pi_{T}} \nu\left(u_{\varepsilon}(t, x)\right) \Delta \psi(t, x) d x d t
\end{aligned}
$$

Let $G$ be the associated Kirchoff's function of $\phi$, given by $G(x)=\int_{0}^{x} \sqrt{\phi^{\prime}(r)} d r$. A simple calculation shows that $\left|\nabla G\left(u_{\varepsilon}(t, x)\right)\right|^{2}=\phi^{\prime}\left(u_{\varepsilon}(t, x)\right)\left|\nabla u_{\varepsilon}(t, x)\right|^{2}$. Since $\beta$ and $\psi$ are nonnegative functions, we obtain

$$
\begin{aligned}
0 & \leq \int_{\mathbb{R}^{d}} \beta\left(u_{\varepsilon}(0, x)\right) \psi(0, x) d x+\int_{\Pi_{T}}\left\{\beta\left(u_{\varepsilon}(t, x)\right) \partial_{t} \psi(t, x)-\nabla \psi(t, x) \cdot \zeta\left(u_{\varepsilon}(t, x)\right)\right\} d x d t \\
& -\int_{\Pi_{T}} \beta^{\prime \prime}\left(u_{\varepsilon}(t, x)\right)\left|\nabla G\left(u_{\varepsilon}(t, x)\right)\right|^{2} \psi(t, x) d x d t+\int_{\Pi_{T}} \nu\left(u_{\varepsilon}(t, x)\right) \Delta \psi(t, x) d x d t+\mathcal{O}(\varepsilon) \\
& +\int_{\Pi_{T}} \int_{E} \int_{0}^{1} \eta\left(x, u_{\varepsilon}(t, x) ; z\right) \beta^{\prime}\left(u_{\varepsilon}(t, x)+\theta \eta\left(x, u_{\varepsilon}(t, x) ; z\right)\right) \psi(t, x) d \theta \tilde{N}(d z, d t) d x \\
& +\int_{\Pi_{T}} \int_{E} \int_{0}^{1}(1-\theta) \eta^{2}\left(x, u_{\varepsilon}(t, x) ; z\right) \beta^{\prime \prime}\left(u_{\varepsilon}(t, x)+\theta \eta\left(x, u_{\varepsilon}(t, x) ; z\right)\right) \psi(t, x) d \theta m(d z) d x d t
\end{aligned}
$$

Clearly, the above inequality is stable under the limit $\varepsilon \rightarrow 0$, if the family $\left\{u_{\varepsilon}\right\}_{\varepsilon>0}$ has $L_{\text {loc }}^{p}$-type stability. Just as the deterministic equations, the above inequality provides us with the entropy condition. We now formally define the entropy solution.

Definition 2.2 (Stochastic entropy solution). A stochastic process $u \in N_{\omega}^{2}\left(0, T, L^{2}\left(\mathbb{R}^{d}\right)\right)$ is called a stochastic entropy solution of (1.1) if 
(1) for each $T>0, G(u) \in L^{2}\left((0, T) \times \Omega ; H^{1}\left(\mathbb{R}^{d}\right)\right)$ and $\sup _{0 \leq t \leq T} \mathbb{E}\left[\|u(t)\|_{2}^{2}\right]<\infty$.

(2) Given a nonnegative test function $\psi \in C_{c}^{1,2}\left([0, \infty) \times \mathbb{R}^{d}\right)$ and a convex entropy flux triple $(\beta, \zeta, \nu)$, the following inequality holds:

$$
\begin{aligned}
& \int_{\Pi_{T}}\left\{\beta(u(t, x)) \partial_{t} \psi(t, x)+\nu(u(t, x)) \Delta \psi(t, x)-\nabla \psi(t, x) \cdot \zeta(u(t, x))\right\} d x d t \\
& +\int_{\Pi_{T}} \int_{E} \int_{0}^{1} \eta(x, u(t, x) ; z) \beta^{\prime}(u(t, x)+\theta \eta(x, u(t, x) ; z)) \psi(t, x) d \theta \tilde{N}(d z, d t) d x \\
& \quad+\int_{\Pi_{T}} \int_{E} \int_{0}^{1}(1-\theta) \eta^{2}(x, u(t, x) ; z) \beta^{\prime \prime}(u(t, x)+\theta \eta(x, u(t, x) ; z)) \psi(t, x) d \theta m(d z) d x d t \\
& \quad \geq \int_{\Pi_{T}} \beta^{\prime \prime}(u(t, x))|\nabla G(u(t, x))|^{2} \psi(t, x) d x d t-\int_{\mathbb{R}^{d}} \beta\left(u_{0}(x)\right) \psi(0, x) d x, \quad P-\text { a.s. }
\end{aligned}
$$

Remark 2.1. We point out that, by a classical separability argument, it is possible to choose a subset of $\Omega$ of $P$-full measure such that (2.3) holds on that subset for every admissible entropy triplet and test function.

The primary aim of this paper is to establish the existence and uniqueness of entropy solutions for the Cauchy problem (1.1) in accordance with Definition 2.2, and we do so under the following assumptions:

(A.1) $\phi: \mathbb{R} \rightarrow \mathbb{R}$ is a non-decreasing Lipschitz continuous function with $\phi(0)=0$. Moreover, if $\eta$ is not a constant function with respect to the space variable $x, t \longmapsto \sqrt{\phi^{\prime}(t)}$ has a modulus of continuity $\omega_{\phi}$ such that $\frac{\omega_{\phi}(r)}{r^{\frac{2}{3}}} \longrightarrow 0$ as $r \rightarrow 0$.

(A.2) $f=\left(f_{1}, f_{2}, \cdots, f_{d}\right): \mathbb{R} \rightarrow \mathbb{R}^{d}$ is a Lipschitz continuous function with $f_{k}(0)=0$ for all $1 \leq k \leq d$.

(A.3) The space $E$ is of the form $\mathcal{O} \times \mathbb{R}^{*}$ and the Borel measure $m$ on $E$ has the form $\lambda \times \mu$ where $\lambda$ is a Radon measure on $\mathcal{O}$ and $\mu$ is a so-called one dimensional Lévy measure.

(A.4) There exist positive constants $K>0, \lambda^{*} \in(0,1)$ and $h_{1}(z) \in L^{2}(E, m)$ with $0 \leq h_{1}(z) \leq 1$ such that

$|\eta(x, u ; z)-\eta(y, v ; z)| \leq\left(\lambda^{*}|u-v|+K|x-y|\right) h_{1}(z)$ for all $x, y \in \mathbb{R}^{d} ; u, v \in \mathbb{R} ; z \in E$.

(A.5) There exists a nonnegative function $g \in L^{\infty}\left(\mathbb{R}^{d}\right) \cap L^{2}\left(\mathbb{R}^{d}\right)$ and $h_{2}(z) \in L^{2}(E, m)$ such that for all $(x, u, z) \in \mathbb{R}^{d} \times \mathbb{R} \times E$,

$$
|\eta(x, u ; z)| \leq g(x)(1+|u|) h_{2}(z) .
$$

The above definition does not say anything explicitly about the entropy solution satisfying the initial condition. However, the initial condition is satisfied in a certain weak sense. Here we state the lemma whose proof follows a simple line argument as in the Lemma 2.3 of [7].

Lemma 2.2. Any entropy solution $u(t, \cdot)$ of (1.1) satisfies the initial condition in the following sense: for every non negative test function $\psi \in C_{c}^{2}\left(\mathbb{R}^{d}\right)$ such that $\operatorname{supp}(\psi)=K$

$$
\lim _{h \rightarrow 0} \mathbb{E}\left[\frac{1}{h} \int_{0}^{h} \int_{K}\left|u(t, x)-u_{0}(x)\right| \psi(x) d x d t\right]=0
$$

Next, we describe a special class of entropy functions that plays an important role in later analysis. Let $\beta: \mathbb{R} \rightarrow \mathbb{R}$ be a $C^{\infty}$ and Lipschitz function satisfying

$$
\beta(0)=0, \quad \beta(-r)=\beta(r), \quad \beta^{\prime \prime} \geq 0,
$$

and

$$
\beta^{\prime}(r)=\left\{\begin{array}{l}
-1 \quad \text { when } r \leq-1 \\
\in[-1,1] \quad \text { when }|r|<1 \\
+1 \quad \text { when } r \geq 1
\end{array}\right.
$$


For any $\vartheta>0$, define $\beta_{\vartheta}: \mathbb{R} \rightarrow \mathbb{R}$ by

$$
\beta_{\vartheta}(r)=\vartheta \beta\left(\frac{r}{\vartheta}\right)
$$

Then

$$
|r|-M_{1} \vartheta \leq \beta_{\vartheta}(r) \leq|r| \quad \text { and } \quad\left|\beta_{\vartheta}^{\prime \prime}(r)\right| \leq \frac{M_{2}}{\vartheta} \mathbf{1}_{|r| \leq \vartheta}
$$

where

$$
M_{1}=\sup _{|r| \leq 1}|| r|-\beta(r)|, \quad M_{2}=\sup _{|r| \leq 1}\left|\beta^{\prime \prime}(r)\right| .
$$

By simply dropping $\vartheta$, for $\beta=\beta_{\vartheta}$ we define

$$
\left\{\begin{array}{l}
\phi^{\beta}(a, b)=\int_{b}^{a} \beta^{\prime}(\sigma-b) \phi^{\prime}(\sigma) d(\sigma), \quad F_{k}^{\beta}(a, b)=\int_{b}^{a} \beta^{\prime}(\sigma-b) f_{k}^{\prime}(\sigma) d(\sigma), \\
F_{k}(a, b)=\operatorname{sign}(a-b)\left(f_{k}(a)-f_{k}(b)\right), \quad F(a, b)=\left(F_{1}(a, b), F_{2}(a, b), \ldots, F_{d}(a, b)\right) .
\end{array}\right.
$$

We conclude this section by stating the main results of this paper.

Theorem 2.3. (Existence) Let the assumptions $(\mathbf{A} .1)(\mathbf{A . 5})$ be true, and that $L^{2}\left(\mathbb{R}^{d}\right)$-valued $\mathcal{F}_{0^{-}}$ measurable random variable $u_{0}$ satisfies $\mathbb{E}\left[\left\|u_{0}\right\|_{2}^{2}<\infty\right.$. Then, there exists an entropy solution of (1.1) in the sense of Definition 2.2

Theorem 2.4. (Uniqueness) Let the assumptions (A.1) (A.5) be true, and that $L^{2}\left(\mathbb{R}^{d}\right)$-valued $\mathcal{F}_{0}$-measurable random variable $u_{0}$ satisfies $\mathbb{E}\left[\left\|u_{0}\right\|_{2}^{2}\right]<\infty$. Then, the entropy solution of (1.1) is unique.

Remark 2.5. In addition, if $u_{0}$ is $L^{p}\left(\mathbb{R}^{d}\right)$ for $p \in[2, \infty)$ then it could be concluded that $u \in$ $L^{\infty}\left(0, T, L^{p}\left(\Omega \times \mathbb{R}^{d}\right)\right)$. Furthermore, if $u_{0} \in L^{\infty}$ and there is $M>0$ such that $\eta(x, u ; z)=0$ for $|u|>M$ and $M_{1}=\sup _{x,|u| \leq M, z}|\eta(x, u ; z)|<\infty$, then $|u(t, x)| \leq \max \left\{M+M_{1},\left\|u_{0}\right\|_{\infty}\right\}$ for almost every $(t, x, \omega) \in \Pi_{T} \times \Omega$. We sketch a justification of this claim in Section 4 .

\section{EXISTENCE OF WEAK SOLUTION FOR VISCOUS PROBLEM}

Just as the deterministic problem, here also we study the corresponding regularized problem by adding a small diffusion operator and derive some a priori bounds. Due to the nonlinear function $\phi$ and related degeneracy, one cannot expect classical solution and instead seeks an weak solution.

3.1. Existence of weak solution to viscous problem. For a small parameter $\varepsilon>0$, we consider the viscous approximation of (1.1) as

$$
\begin{gathered}
d u(t, x)-\Delta \phi(u(t, x)) d t=\operatorname{div}_{x} f(u(t, x)) d t+\int_{E} \eta(x, u(t, x) ; z) \tilde{N}(d z, d t) \\
+\varepsilon \Delta u(t, x) d t, \quad t>0, x \in \mathbb{R}^{d} .
\end{gathered}
$$

In this subsection, we establish the existence of a weak solution for the problem (3.1). To do this, we use an implicit time discretization scheme. Let $\Delta t=\frac{T}{N}$ for some positive integer $N \geq 1$. Set $t_{n}=n \Delta t$ for $n=0,1,2 \cdots, N$.

Define

$$
\begin{array}{cl}
\mathcal{N}=L^{2}\left(\Omega ; H^{1}\left(\mathbb{R}^{d}\right)\right), & \mathcal{N}_{n}=\left\{\text { the } \mathcal{F}_{n \Delta t} \text { measurable elements of } \mathcal{N}\right\}, \\
\mathcal{H}=L^{2}\left(\Omega ; L^{2}\left(\mathbb{R}^{d}\right)\right), & \mathcal{H}_{n}=\left\{\text { the } \mathcal{F}_{n \Delta t} \text { measurable elements of } \mathcal{H}\right\} .
\end{array}
$$

Proposition 3.1. Assume that $\Delta t$ is small. For any given $u_{n} \in \mathcal{H}_{n}$, there exists a unique $u_{n+1} \in$ $\mathcal{N}_{n+1}$ with $\phi\left(u_{n+1}\right) \in \mathcal{N}_{n+1}$ such that $P-$ a.s. for any $v \in H^{1}\left(\mathbb{R}^{d}\right)$, the following variational formula holds:

$$
\begin{aligned}
& \int_{\mathbb{R}^{d}}\left(\left(u_{n+1}-u_{n}\right) v+\Delta t\left\{\nabla \phi\left(u_{n+1}\right)+\varepsilon \nabla u_{n+1}+f\left(u_{n+1}\right)\right\} \cdot \nabla v\right) d x \\
& =\int_{\mathbb{R}^{d}} \int_{t_{n}}^{t_{n+1}} \int_{E} \eta\left(x, u_{n} ; z\right) v \tilde{N}(d z, d s) d x .
\end{aligned}
$$


Before proving the proposition, first we state a key deterministic lemma, related to the weak solution of degenerate parabolic equations. We have the following lemma, a proof of which could be found in [ page 19, 9] ].

Lemma 3.2. Assume that $\Delta t$ is small and $X \in L^{2}\left(\mathbb{R}^{d}\right)$. Then, for fixed positive parameter $\varepsilon>0$,

(1) there exists a unique $u \in H^{1}\left(\mathbb{R}^{d}\right)$ with $\phi(u) \in H^{1}\left(\mathbb{R}^{d}\right)$ such that, for any $v \in H^{1}\left(\mathbb{R}^{d}\right)$

$$
\int_{\mathbb{R}^{d}}(u v+\Delta t\{\nabla \phi(u)+\varepsilon \nabla u+f(u)\} \cdot \nabla v) d x=\int_{\mathbb{R}^{d}} X v d x .
$$

(2) There exists a constant $C=C(\Delta t)>0$ such that the following a priori estimate holds

$$
\|u\|_{L^{2}\left(\mathbb{R}^{d}\right)}^{2}+\|\phi(u)\|_{H^{1}\left(\mathbb{R}^{d}\right)}^{2}+\varepsilon\|\nabla u\|_{L^{2}\left(\mathbb{R}^{d}\right)}^{2} \leq C\|X\|_{L^{2}\left(\mathbb{R}^{d}\right)}^{2} .
$$

(3) The map $\Theta: X \in L^{2}\left(\mathbb{R}^{d}\right) \mapsto(u, \phi(u)) \in H^{1}\left(\mathbb{R}^{d}\right)^{2}$ is continuous.

Proof of the Proposition 3.1 Let $u_{n} \in \mathcal{N}_{n}$. Take $X=u_{n}+\int_{t_{n}}^{t_{n+1}} \int_{E} \eta\left(x, u_{n} ; z\right) \tilde{N}(d z, d s)$. Then, by the assumption (A.5) we obtain

$$
\mathbb{E}\left[\|X\|_{L^{2}\left(\mathbb{R}^{d}\right)}^{2}\right] \leq\left\|u_{n}\right\|_{\mathcal{H}}^{2}+C \Delta t\left(\|g\|_{L^{2}\left(\mathbb{R}^{d}\right)}^{2}+\left\|u_{n}\right\|_{\mathcal{H}}^{2}\right) .
$$

This shows that for a.s. $\omega \in \Omega, X \in L^{2}\left(\mathbb{R}^{d}\right)$. Therefore, one can use the Lemma 3.2. and conclude that for almost surely $\omega \in \Omega$, there exist unique $u(\omega)$ satisfying the variational equality (3.2). Moreover, by construction $X \in \mathcal{H}_{n+1}$. Thus, due to the continuity of $\Theta$ for the $\mathcal{F}_{(n+1) \Delta t}$ measurability and to a priori estimate (3.4), we conclude that $u \in \mathcal{N}_{n+1}$ with $\phi(u) \in \mathcal{N}_{n+1}$. We denote this solution $u$ by $u_{n+1}$. Hence the proof of the proposition follows.

3.1.1. A priori estimate. Note that, for any $v \in \mathcal{D}\left(\mathbb{R}^{d}\right), \int_{\mathbb{R}^{d}} f(v) \cdot \nabla v d x=0$ and hence true for any $v \in H^{1}\left(\mathbb{R}^{d}\right)$ by density argument. We choose a test function $v=u_{n+1}$ in (3.2) and have

$$
\begin{aligned}
& \int_{\mathbb{R}^{d}}\left(u_{n+1}-u_{n}\right) u_{n+1} d x+\Delta t \int_{\mathbb{R}^{d}} \phi^{\prime}\left(u_{n+1}\right)\left|\nabla u_{n+1}\right|^{2} d x+\varepsilon \Delta t \int_{\mathbb{R}^{d}}\left|\nabla u_{n+1}\right|^{2} d x \\
& =\int_{\mathbb{R}^{d}} \int_{t_{n}}^{t_{n+1}} \int_{E} \eta\left(x, u_{n} ; z\right) \tilde{N}(d z, d s) u_{n+1} d x \\
& \leq \int_{\mathbb{R}^{d}} \int_{t_{n}}^{t_{n+1}} \int_{E} \eta\left(x, u_{n} ; z\right) u_{n} \tilde{N}(d z, d s) d x+\frac{\alpha}{2}\left\|u_{n+1}-u_{n}\right\|_{L^{2}\left(\mathbb{R}^{d}\right)}^{2} \\
& \quad+\frac{1}{2 \alpha} \int_{\mathbb{R}^{d}}\left(\int_{t_{n}}^{t_{n+1}} \int_{E} \eta\left(x, u_{n} ; z\right) \tilde{N}(d z, d s)\right)^{2} d x, \quad \text { for some } \alpha>0 .
\end{aligned}
$$

Since $\int_{\mathbb{R}^{d}}|\nabla \phi(u)|^{2} d x=\int_{\mathbb{R}^{d}}\left|\phi^{\prime}(u) \nabla u\right|^{2} d x \leq c_{\phi} \int_{\mathbb{R}^{d}} \phi^{\prime}(u)|\nabla u|^{2} d x$, we see that

$$
\frac{\Delta t}{c_{\phi}}\|\nabla \phi(u)\|_{\mathcal{H}}^{2} \leq \Delta t \mathbb{E}\left[\int_{\mathbb{R}^{d}} \phi^{\prime}(u)|\nabla u|^{2} d x\right] .
$$

In view of the assumption (A.5) the inequality (3.5), Itô-Lévy isometry, and the fact that for any $a, b \in \mathbb{R},(a-b) a=\frac{1}{2}\left(a^{2}+(a-b)^{2}-b^{2}\right)$, we obtain

$$
\begin{aligned}
& \frac{1}{2}\left[\left\|u_{n+1}\right\|_{\mathcal{H}}^{2}+\left\|u_{n+1}-u_{n}\right\|_{\mathcal{H}}^{2}-\left\|u_{n}\right\|_{\mathcal{H}}^{2}\right]+\frac{\Delta t}{c_{\phi}}\left\|\nabla \phi\left(u_{n+1}\right)\right\|_{\mathcal{H}}^{2}+\varepsilon \Delta t\left\|\nabla u_{n+1}\right\|_{\mathcal{H}}^{2} \\
& \leq \frac{\alpha}{2}\left\|u_{n+1}-u_{n}\right\|_{\mathcal{H}}^{2}+\frac{C \Delta t}{2 \alpha}\left(1+\left\|u_{n}\right\|_{\mathcal{H}}^{2}\right) .
\end{aligned}
$$

Since $\alpha>0$ is arbitrary, one can choose $\alpha>0$ so that

$$
\begin{aligned}
& \left\|u_{n}\right\|_{\mathcal{H}}^{2}+\sum_{k=0}^{n-1}\left\|u_{k+1}-u_{k}\right\|_{\mathcal{H}}^{2}+\frac{\Delta t}{c_{\phi}} \sum_{k=0}^{n-1}\left\|\nabla \phi\left(u_{k+1}\right)\right\|_{\mathcal{H}}^{2}+\varepsilon \Delta t \sum_{k=0}^{n-1}\left\|\nabla u_{k+1}\right\|_{\mathcal{H}}^{2} \\
& \leq C_{1}+C_{2} \Delta t \sum_{k=0}^{n-1}\left\|u_{k}\right\|_{\mathcal{H}}^{2}, \quad \text { for some constants } C_{1}, C_{2}>0 .
\end{aligned}
$$


Thanks to discrete Gronwall's lemma, one has from (3.6),

$$
\left\|u_{n}\right\|_{\mathcal{H}}^{2}+\sum_{k=0}^{n-1}\left\|u_{k+1}-u_{k}\right\|_{\mathcal{H}}^{2}+\frac{\Delta t}{c_{\phi}} \sum_{k=0}^{n-1}\left\|\nabla \phi\left(u_{k+1}\right)\right\|_{\mathcal{H}}^{2}+\varepsilon \Delta t \sum_{k=0}^{n-1}\left\|\nabla u_{k+1}\right\|_{\mathcal{H}}^{2} \leq C
$$

For fixed $\Delta t=\frac{T}{N}$, we define

$u^{\Delta t}(t)=\sum_{k=1}^{N} u_{k} \mathbf{1}_{[(k-1) \Delta t, k \Delta t)}(t) ; \quad \tilde{u}^{\Delta t}(t)=\sum_{k=1}^{N}\left[\frac{u_{k}-u_{k-1}}{\Delta t}(t-(k-1) \Delta t)+u_{k-1}\right] \mathbf{1}_{[(k-1) \Delta t, k \Delta t)}(t)$ with $u^{\Delta t}(t)=u_{0}$ for $t<0$. Similarly, we define

$$
\tilde{B}^{\Delta t}(t)=\sum_{k=1}^{N}\left[\frac{B_{k}-B_{k-1}}{\Delta t}[t-(k-1) \Delta t]+B_{k-1}\right] \mathbf{1}_{[(k-1) \Delta t, k \Delta t)}(t)
$$

where

$$
B_{n}=\sum_{k=0}^{n-1} \int_{k \Delta t}^{(k+1) \Delta t} \int_{E} \eta\left(x, u_{k} ; z\right) \tilde{N}(d z, d s)=\int_{0}^{n \Delta t} \int_{E} \eta\left(x, u^{\Delta t}(s-\Delta t) ; z\right) \tilde{N}(d z, d s) .
$$

A straightforward calculation shows that

$$
\left\{\begin{array}{l}
\left\|u^{\Delta t}\right\|_{L^{\infty}(0, T ; \mathcal{H})}=\max _{k=1,2, \cdots, N}\left\|u_{k}\right\|_{\mathcal{H}} ; \quad\left\|\tilde{u}^{\Delta t}\right\|_{L^{\infty}(0, T ; \mathcal{H})}=\max _{k=0,1, \cdots, N}\left\|u_{k}\right\|_{\mathcal{H}}, \\
\left\|u^{\Delta t}-\tilde{u}^{\Delta t}\right\|_{L^{2}(0, T ; \mathcal{H})}^{2} \leq \Delta t \sum_{k=0}^{N-1}\left\|u_{k+1}-u_{k}\right\|_{\mathcal{H}^{*}}^{2}
\end{array}\right.
$$

Since $\phi$ is a Lipschitz continuous function with $\phi(0)=0$, in view of the above definitions and $a$ priori estimate (3.7), we have the following proposition.

Proposition 3.3. Assume that $\Delta t$ is small. Then $u^{\Delta t}, \tilde{u}^{\Delta t}$ are bounded sequences in $L^{\infty}(0, T ; \mathcal{H})$; $\phi\left(u^{\Delta t}\right), \sqrt{\epsilon} u^{\Delta t}$ are a bounded sequences in $L^{2}(0, T ; \mathcal{N})$ and $\left\|u^{\Delta t}-\tilde{u}^{\Delta t}\right\|_{L^{2}(0, T ; \mathcal{H})}^{2} \leq C \Delta t$.

Moreover, $u^{\Delta t}-u^{\Delta t}(\cdot-\Delta t) \rightarrow 0$ in $L^{2}\left(\Omega \times \Pi_{T}\right)$.

Next, we want to find some upper bound for $\tilde{B}^{\Delta t}(t)$. Regarding this, we have the following proposition.

Proposition 3.4. $\tilde{B}^{\Delta t}$ is a bounded sequence in $L^{2}\left(\Omega \times \Pi_{T}\right)$ and

$$
\left\|\tilde{B}^{\Delta t}(\cdot)-\int_{0}^{\cdot} \int_{E} \eta\left(x, u^{\Delta t}(s-\Delta t) ; z\right) \tilde{N}(d z, d s)\right\|_{L^{2}\left(\Omega \times \mathbb{R}^{d}\right)}^{2} \leq C \Delta t .
$$

Proof. First we prove the boundedness of $\tilde{B}^{\Delta t}(t)$. By using definition of $\tilde{B}^{\Delta t}(t)$, the assumption $(\mathbf{A} .5)$ and boundedness of $u^{\Delta t}$ in $L^{\infty}(0, T ; \mathcal{H})$, we obtain

$$
\begin{aligned}
\left\|\tilde{B}^{\Delta t}\right\|_{L^{2}\left(0, T ; L^{2}\left(\Omega, L^{2}\left(\mathbb{R}^{d}\right)\right)\right)}^{2} & \leq \Delta t \sum_{k=0}^{N}\left\|B_{k}\right\|_{L^{2}\left(\Omega \times \mathbb{R}^{d}\right)}^{2} \\
& \leq \Delta t \sum_{k=0}^{N} \mathbb{E}\left[\left|\int_{\mathbb{R}^{d}} \int_{0}^{k \Delta t} \int_{E} \eta\left(x, u^{\Delta t}(s-\Delta t) ; z\right) \tilde{N}(d z, d s) d x\right|^{2}\right] \\
& \leq C \Delta t \sum_{k=0}^{N} \mathbb{E}\left[\int_{\mathbb{R}^{d}} \int_{0}^{k \Delta t} g^{2}(x)\left(1+\left|u^{\Delta t}(s-\Delta t)\right|^{2}\right) d x d s\right] \\
& \leq C\left(1+\left\|u^{\Delta t}\right\|_{L^{\infty}\left(0, T ; L^{2}\left(\Omega \times \mathbb{R}^{d}\right)\right)}\right)<+\infty .
\end{aligned}
$$

Thus, $\tilde{B}^{\Delta t}$ is a bounded sequence in $L^{2}\left(\Omega \times \Pi_{T}\right)$.

To prove second part of the proposition, we see that for any $t \in[n \Delta t,(n+1) \Delta t)$,

$$
\tilde{B}^{\Delta t}(t)-\int_{0}^{t} \int_{E} \eta\left(x, u^{\Delta t}(s-\Delta t) ; z\right) \tilde{N}(d z, d s)
$$




$$
\begin{aligned}
& =\frac{t-n \Delta t}{\Delta t} \int_{n \Delta t}^{(n+1) \Delta t} \int_{E} \eta\left(x, u^{\Delta t}(s-\Delta t) ; z\right) \tilde{N}(d z, d s)-\int_{n \Delta t}^{t} \int_{E} \eta\left(x, u^{\Delta t}(s-\Delta t) ; z\right) \tilde{N}(d z, d s) \\
& =\frac{t-n \Delta t}{\Delta t} \int_{n \Delta t}^{(n+1) \Delta t} \int_{E} \eta\left(x, u_{n} ; z\right) \tilde{N}(d z, d s)-\int_{n \Delta t}^{t} \int_{E} \eta\left(x, u_{n} ; z\right) \tilde{N}(d z, d s) .
\end{aligned}
$$

Therefore, in view of (3.7) and assumption (A.5), we have

$$
\begin{aligned}
& \left\|\tilde{B}^{\Delta t}(t)-\int_{0}^{t} \int_{E} \eta\left(x, u^{\Delta t}(s-\Delta t) ; z\right) \tilde{N}(d z, d s)\right\|_{L^{2}\left(\Omega \times \mathbb{R}^{d}\right)}^{2} \\
= & \mathbb{E}\left[\int_{\mathbb{R}^{d}}\left|\frac{t-n \Delta t}{\Delta t} \int_{n \Delta t}^{(n+1) \Delta t} \int_{E} \eta\left(x, u_{n} ; z\right) \tilde{N}(d z, d s)-\int_{n \Delta t}^{t} \int_{E} \eta\left(x, u_{n} ; z\right) \tilde{N}(d z, d s)\right|^{2} d x\right] \\
\leq & 2 \int_{\mathbb{R}^{d}} \mathbb{E}\left[\left(\frac{t-n \Delta t}{\Delta t}\right)^{2} \int_{n \Delta t}^{(n+1) \Delta t} \int_{E} \eta^{2}\left(x, u_{n} ; z\right) m(d z) d s+\int_{n \Delta t}^{t} \int_{E} \eta^{2}\left(x, u_{n} ; z\right) m(d z) d s\right] d x \\
\leq & C\left(1+\left\|u_{n}\right\|_{\mathcal{H}}^{2}\right)\left[\frac{(t-n \Delta t)^{2}}{\Delta t}+(t-n \Delta t)\right] \leq C \Delta t .
\end{aligned}
$$

This completes the proof.

3.1.2. Convergence of $u^{\Delta t}(t, x)$. Thanks to Proposition 3.3 and Lipschitz property of $f$ and $\phi$, there exist $u, \phi_{u}$ and $f_{u}$ such that (up to a subsequence)

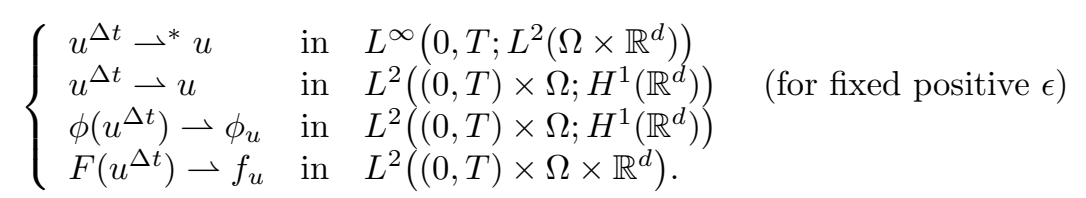

Next, we want to identify the weak limits $\phi_{u}$ and $f_{u}$. Note that, for any $v \in H^{1}\left(\mathbb{R}^{d}\right)$, we can rewrite (3.2), in terms of $u^{\Delta t}, \tilde{u}^{\Delta t}$ and $\tilde{B}^{\Delta t}$ as

$$
\int_{\mathbb{R}^{d}}\left(\frac{\partial}{\partial t}\left(\tilde{u}^{\Delta t}-\tilde{B}^{\Delta t}\right)(t) v+\left\{\nabla \phi\left(u^{\Delta t}(t)\right)+\varepsilon \nabla u^{\Delta t}(t)+f\left(u^{\Delta t}(t)\right)\right\} \cdot \nabla v\right) d x=0 .
$$

Lemma 3.5. $\left\{u^{\Delta t}\right\}$ is a Cauchy sequence in $L^{2}\left(\Omega \times \Pi_{T}\right)$.

Proof. Consider two positive integers $N$ and $M$ and denote $\Delta t=\frac{T}{N}, \Delta s=\frac{T}{M}$. Then, for any $v \in H^{1}\left(\mathbb{R}^{d}\right)$, one gets from (3.9),

$$
\begin{aligned}
& \int_{\mathbb{R}^{d}}\left(\frac{\partial}{\partial t}\left[\left(\tilde{u}^{\Delta t}-\tilde{B}^{\Delta t}\right)(t)-\left(\tilde{u}^{\Delta s}-\tilde{B}^{\Delta s}\right)(t)\right] v+\left\{\nabla\left(\phi\left(u^{\Delta t}(t)\right)-\phi\left(u^{\Delta s}(t)\right)\right)\right.\right. \\
& \left.\left.+\varepsilon \nabla\left(u^{\Delta t}(t)-u^{\Delta s}(t)\right)+\left(f\left(u^{\Delta t}(t)\right)-f\left(u^{\Delta s}(t)\right)\right)\right\} \cdot \nabla v\right) d x=0 .
\end{aligned}
$$

Let $w=\left(\tilde{u}^{\Delta t}-\tilde{B}^{\Delta t}\right)(t)-\left(\tilde{u}^{\Delta s}-\tilde{B}^{\Delta s}\right)(t)$. Set $v=(I-\Delta)^{-1} w$ in (3.10). Then, one has

$$
\begin{aligned}
& \frac{1}{2} \frac{\partial}{\partial t}\|v(t)\|_{H^{1}\left(\mathbb{R}^{d}\right)}^{2}+\int_{\mathbb{R}^{d}}\left(\phi\left(u^{\Delta t}(t)\right)-\phi\left(u^{\Delta s}(t)\right)\right) w d x-\int_{\mathbb{R}^{d}}\left(\phi\left(u^{\Delta t}(t)\right)-\phi\left(u^{\Delta s}(t)\right)\right) v d x \\
& +\varepsilon \int_{\mathbb{R}^{d}}\left(u^{\Delta t}-u^{\Delta s}\right) w d x-\varepsilon \int_{\mathbb{R}^{d}}\left(u^{\Delta t}-u^{\Delta s}\right) v d x+\int_{\mathbb{R}^{d}}\left(f\left(u^{\Delta t}\right)-f\left(u^{\Delta s}\right)\right) \cdot \nabla v d x=0 .
\end{aligned}
$$

Note that, $w=\left(u^{\Delta t}-u^{\Delta s}\right)-\left(\tilde{B}^{\Delta t}-\tilde{B}^{\Delta s}\right)-\left(u^{\Delta t}-\tilde{u}^{\Delta t}\right)+\left(u^{\Delta s}-\tilde{u}^{\Delta s}\right)$.

Therefore,

$$
\begin{aligned}
& \int_{\mathbb{R}^{d}}\left(\phi\left(u^{\Delta t}(t)\right)-\phi\left(u^{\Delta s}(t)\right)\right) w d x \\
= & \int_{\mathbb{R}^{d}}\left(\phi\left(u^{\Delta t}(t)\right)-\phi\left(u^{\Delta s}(t)\right)\right)\left(u^{\Delta t}-u^{\Delta s}\right) d x-\int_{\mathbb{R}^{d}}\left(\phi\left(u^{\Delta t}(t)\right)-\phi\left(u^{\Delta s}(t)\right)\right)\left(\tilde{B}^{\Delta t}-\tilde{B}^{\Delta s}\right) d x \\
& -\int_{\mathbb{R}^{d}}\left(\phi\left(u^{\Delta t}(t)\right)-\phi\left(u^{\Delta s}(t)\right)\right)\left\{\left(u^{\Delta t}-\tilde{u}^{\Delta t}\right)+\left(\tilde{u}^{\Delta s}-u^{\Delta s}\right)\right\} d x
\end{aligned}
$$




$$
\begin{aligned}
& \geq \int_{\mathbb{R}^{d}}\left(\phi\left(u^{\Delta t}(t)\right)-\phi\left(u^{\Delta s}(t)\right)\right)\left(u^{\Delta t}-u^{\Delta s}\right) d x-\frac{1}{2 c_{\phi}} \int_{\mathbb{R}^{d}}\left(\phi\left(u^{\Delta t}(t)\right)-\phi\left(u^{\Delta s}(t)\right)\right)^{2} d x \\
& -\frac{c_{\phi}}{2} \int_{\mathbb{R}^{d}}\left\{\left(\tilde{B}^{\Delta t}-\tilde{B}^{\Delta s}\right)+\left(u^{\Delta t}-\tilde{u}^{\Delta t}\right)+\left(\tilde{u}^{\Delta s}-u^{\Delta s}\right)\right\}^{2} d x \\
& \geq \frac{1}{2} \int_{\mathbb{R}^{d}}\left(\phi\left(u^{\Delta t}(t)\right)-\phi\left(u^{\Delta s}(t)\right)\right)\left(u^{\Delta t}-u^{\Delta s}\right) d x \\
& -\frac{c_{\phi}}{2} \int_{\mathbb{R}^{d}}\left\{\left(\tilde{B}^{\Delta t}-\tilde{B}^{\Delta s}\right)+\left(u^{\Delta t}-\tilde{u}^{\Delta t}\right)+\left(\tilde{u}^{\Delta s}-u^{\Delta s}\right)\right\}^{2} d x \\
& \geq-\frac{c_{\phi}}{2} \int_{\mathbb{R}^{d}}\left\{\left(\tilde{B}^{\Delta t}-\tilde{B}^{\Delta s}\right)+\left(u^{\Delta t}-\tilde{u}^{\Delta t}\right)+\left(\tilde{u}^{\Delta s}-u^{\Delta s}\right)\right\}^{2} d x \quad \text { (by ex (A.1) } \text {. }
\end{aligned}
$$

Similarly, we also have

$$
\begin{aligned}
& \varepsilon \int_{\mathbb{R}^{d}}\left(u^{\Delta t}-u^{\Delta s}\right) w d x \\
\geq & \frac{\varepsilon}{2} \int_{\mathbb{R}^{d}}\left(u^{\Delta t}-u^{\Delta s}\right)^{2} d x-\frac{\varepsilon}{2} \int_{\mathbb{R}^{d}}\left\{\left(\tilde{B}^{\Delta t}-\tilde{B}^{\Delta s}\right)+\left(u^{\Delta t}-\tilde{u}^{\Delta t}\right)+\left(\tilde{u}^{\Delta s}-u^{\Delta s}\right)\right\}^{2} d x .
\end{aligned}
$$

Combining (3.11), (3.12) and (3.13) in

$$
\begin{aligned}
& \frac{1}{2} \frac{\partial}{\partial t}\|v(t)\|_{H^{1}\left(\mathbb{R}^{d}\right)}^{2}+\frac{\varepsilon}{2} \int_{\mathbb{R}^{d}}\left(u^{\Delta t}-u^{\Delta s}\right)^{2} d x \\
\leq & \frac{\left(c_{\phi}+\varepsilon\right)}{2} \int_{\mathbb{R}^{d}}\left\{\left(\tilde{B}^{\Delta t}-\tilde{B}^{\Delta s}\right)+\left(u^{\Delta t}-\tilde{u}^{\Delta t}\right)+\left(\tilde{u}^{\Delta s}-u^{\Delta s}\right)\right\}^{2} d x \\
& +\varepsilon \int_{\mathbb{R}^{d}}\left(u^{\Delta t}-u^{\Delta s}\right) v d x-\int_{\mathbb{R}^{d}}\left(F\left(u^{\Delta t}\right)-F\left(u^{\Delta s}\right)\right) \cdot \nabla v d x+\int_{\mathbb{R}^{d}}\left(\phi\left(u^{\Delta t}(t)\right)-\phi\left(u^{\Delta s}(t)\right)\right) v d x \\
\leq & \frac{\left(c_{\phi}+\varepsilon\right)}{2} \int_{\mathbb{R}^{d}}\left\{\left(\tilde{B}^{\Delta t}-\tilde{B}^{\Delta s}\right)+\left(u^{\Delta t}-\tilde{u}^{\Delta t}\right)+\left(\tilde{u}^{\Delta s}-u^{\Delta s}\right)\right\}^{2} d x+\frac{\beta}{4} \int_{\mathbb{R}^{d}}|\nabla v|^{2} d x \\
+ & \frac{\varepsilon}{2 \alpha} \int_{\mathbb{R}^{d}}\left(u^{\Delta t}-u^{\Delta s}\right)^{2} d x+\left(\frac{\beta}{4}+\varepsilon \frac{\alpha}{2}\right) \int_{\mathbb{R}^{d}} v^{2} d x+\frac{\left(c_{f}\right)^{2}+\left(c_{\phi}\right)^{2}}{\beta} \int_{\mathbb{R}^{d}}\left(u^{\Delta t}-u^{\Delta s}\right)^{2} d x
\end{aligned}
$$

for some $\alpha$ and $\beta>0$. Since $\alpha, \beta>0$ are arbitrary, there exist positive constants $C_{1}, C_{2}$ and $C_{3}$ such that

$$
\begin{aligned}
& \mathbb{E}\left[\|v(t)\|_{H^{1}\left(\mathbb{R}^{d}\right)}^{2}\right]-C_{1} \int_{0}^{t} \mathbb{E}\left[\|v(r)\|_{H^{1}\left(\mathbb{R}^{d}\right)}^{2}\right] d r+C_{2} \int_{0}^{t} \mathbb{E}\left[\left\|u^{\Delta t}-u^{\Delta s}\right\|_{L^{2}\left(\mathbb{R}^{d}\right)}^{2}\right] d r \\
& \leq C_{3}\left\{\left\|u^{\Delta t}-\tilde{u}^{\Delta t}\right\|_{L^{2}\left(\Omega \times \Pi_{T}\right)}^{2}+\left\|u^{\Delta s}-\tilde{u}^{\Delta s}\right\|_{L^{2}\left(\Omega \times \Pi_{T}\right)}^{2}+\int_{0}^{t} \mathbb{E}\left[\left\|\tilde{B}^{\Delta t}-\tilde{B}^{\Delta s}\right\|_{L^{2}\left(\mathbb{R}^{d}\right)}^{2}\right] d r\right\} .
\end{aligned}
$$

In view of the Proposition 3.3 we notice that

$$
\left\|u^{\Delta t}-\tilde{u}^{\Delta t}\right\|_{L^{2}\left(\Omega \times \Pi_{T}\right)}^{2}+\left\|u^{\Delta s}-\tilde{u}^{\Delta s}\right\|_{L^{2}\left(\Omega \times \Pi_{T}\right)}^{2} \leq C(\Delta t+\Delta s) .
$$

So we need to estimate the term $\int_{0}^{t} \mathbb{E}\left[\left\|\tilde{B}^{\Delta t}-\tilde{B}^{\Delta s}\right\|_{L^{2}\left(\mathbb{R}^{d}\right)}^{2}\right] d r$. Now

$$
\begin{aligned}
& \mathbb{E}\left[\left\|\tilde{B}^{\Delta t}(r)-\tilde{B}^{\Delta s}(r)\right\|_{L^{2}\left(\mathbb{R}^{d}\right)}^{2}\right] \\
\leq & 3\left\|\tilde{B}^{\Delta t}(r)-\int_{0}^{r} \int_{E} \eta\left(x, u^{\Delta t}(\sigma-\Delta t) ; z\right) \tilde{N}(d z, d \sigma)\right\|_{L^{2}\left(\Omega \times \mathbb{R}^{d}\right)}^{2} \\
& +3\left\|\tilde{B}^{\Delta s}(r)-\int_{0}^{r} \int_{E} \eta\left(x, u^{\Delta s}(\sigma-\Delta s) ; z\right) \tilde{N}(d z, d \sigma)\right\|_{L^{2}\left(\Omega \times \mathbb{R}^{d}\right)}^{2} \\
& +3\left\|\int_{0}^{r} \int_{E}\left(\eta\left(x, u^{\Delta t}(\sigma-\Delta t) ; z\right)-\eta\left(x, u^{\Delta s}(\sigma-\Delta s) ; z\right)\right) \tilde{N}(d z, d \sigma)\right\|_{L^{2}\left(\Omega \times \mathbb{R}^{d}\right)}^{2} \\
\leq & C(\Delta t+\Delta s)+C \int_{0}^{r} \mathbb{E}\left[\left\|u^{\Delta t}(\sigma-\Delta t)-u^{\Delta s}(\sigma-\Delta s)\right\|_{L^{2}\left(\mathbb{R}^{d}\right)}^{2}\right] d \sigma,
\end{aligned}
$$


where we have used Proposition 3.4 and the assumption (A.5) Thus, we get

$$
\begin{aligned}
& \int_{0}^{t} \mathbb{E}\left[\left\|\tilde{B}^{\Delta t}-\tilde{B}^{\Delta s}\right\|_{L^{2}\left(\mathbb{R}^{d}\right)}^{2}\right] d r \\
\leq & C(\Delta t+\Delta s)+C \int_{0}^{t} \int_{0}^{r} \mathbb{E}\left[\left\|u^{\Delta t}(\sigma-\Delta t)-u^{\Delta s}(\sigma-\Delta s)\right\|_{L^{2}\left(\mathbb{R}^{d}\right)}^{2}\right] d \sigma d r .
\end{aligned}
$$

We combine (3.15) and (3.16) in (3.14) and have

$$
\begin{aligned}
& \mathbb{E}\left[\|v(t)\|_{H^{1}\left(\mathbb{R}^{d}\right)}^{2}\right]-C_{1} \int_{0}^{t} \mathbb{E}\left[\|v(r)\|_{H^{1}\left(\mathbb{R}^{d}\right)}^{2}\right] d r+C_{2} \int_{0}^{t} \mathbb{E}\left[\left\|u^{\Delta t}-u^{\Delta s}\right\|_{L^{2}\left(\mathbb{R}^{d}\right)}^{2}\right] d r \\
& \leq C(\Delta t+\Delta s)+C \int_{0}^{t} \int_{0}^{r} \mathbb{E}\left[\left\|u^{\Delta t}(\sigma-\Delta t)-u^{\Delta s}(\sigma-\Delta s)\right\|_{L^{2}\left(\mathbb{R}^{d}\right)}^{2}\right] d \sigma d r
\end{aligned}
$$

(by Proposition 3.3)

$$
\leq C(\Delta t+\Delta s)+C \int_{0}^{t} \int_{0}^{r} \mathbb{E}\left[\left\|u^{\Delta t}-u^{\Delta s}\right\|_{L^{2}\left(\mathbb{R}^{d}\right)}^{2}\right] d \sigma d r .
$$

Hence, an application of the Gronwall's lemma gives

$$
\mathbb{E}\left[\|v(t)\|_{H^{1}\left(\mathbb{R}^{d}\right)}^{2}\right]+\int_{0}^{t} \mathbb{E}\left[\left\|u^{\Delta t}-u^{\Delta s}\right\|_{L^{2}\left(\mathbb{R}^{d}\right)}^{2}\right] d r \leq C(\Delta t+\Delta s) e^{C t} .
$$

This implies that

$$
\left\|u^{\Delta t}-u^{\Delta s}\right\|_{L^{2}\left(\Omega \times \Pi_{T}\right)}^{2} \leq C(\Delta t+\Delta s) e^{C T}
$$

i.e., $\left\{u^{\Delta t}\right\}$ is a Cauchy sequence in $L^{2}\left(\Omega \times \Pi_{T}\right)$.

We are now in a position to identify the weak limits $\phi_{u}$ and $f_{u}$. We have shown that $u^{\Delta t} \rightarrow u$ and $u^{\Delta t}$ is a Cauchy sequence in $L^{2}\left(\Omega \times \Pi_{T}\right)$. Thanks to the Lipschitz continuity of $\phi$ and $f$, one can easily conclude that $\phi_{u}=\phi(u)$ and $f_{u}=f(u)$.

In view of the variational formula (3.9), one needs to show the boundedness of $\frac{\partial}{\partial t}\left(\tilde{u}^{\Delta t}-\tilde{B}^{\Delta t}\right)$ in $L^{2}\left(\Omega \times(0, T) ; H^{-1}\left(\mathbb{R}^{d}\right)\right)$ and then identify the weak limit. Regarding this, we have the following lemma.

Lemma 3.6. The sequence $\left\{\frac{\partial}{\partial t}\left(\tilde{u}^{\Delta t}-\tilde{B}^{\Delta t}\right)(t)\right\}$ is bounded in $L^{2}\left(\Omega \times(0, T) ; H^{-1}\left(\mathbb{R}^{d}\right)\right)$, and

$$
\frac{\partial}{\partial t}\left(\tilde{u}^{\Delta t}-\tilde{B}^{\Delta t}\right) \rightarrow \frac{\partial}{\partial t}\left(u-\int_{0} \int_{E} \eta(x, u ; z) \tilde{N}(d z, d s)\right) \quad \text { in } L^{2}\left(\Omega \times(0, T) ; H^{-1}\left(\mathbb{R}^{d}\right)\right)
$$

where $u$ is given by (3.8).

Proof. To prove the lemma, let $\Gamma=\Omega \times[0, T] \times E, \mathcal{G}=\mathcal{P}_{T} \times \mathcal{L}(E)$ and $\varsigma=P \otimes \ell_{t} \otimes m$, where $\mathcal{P}_{T}$ represents predictable $\sigma$-algebra on $\Omega \times[0, T]$ and $\mathcal{L}(E)$ represents a Lebesgue $\sigma$-algebra on $E$. Then $L^{2}((\Gamma, \mathcal{G}, \varsigma) ; \mathbb{R})$ consists of all square integrable predictable processes which are Borel measurable functions of $z$-variable.

The space $L^{2}((\Gamma, \mathcal{G}, \varsigma) ; \mathbb{R})$ represents the space of square integrable predictable integrands for Itô-Lévy integrals with respect to the compensated compound Poisson random measure $\tilde{N}(d z, d t)$. Moreover, Itô-Lévy integral defines a linear operator from $L^{2}((\Gamma, \mathcal{G}, \varsigma) ; \mathbb{R})$ to $L^{2}\left(\left(\Omega, \mathcal{F}_{T}\right) ; \mathbb{R}\right)$ and it preserves the norm (cf. for example 22]).

Thank to Propositions 3.3 and 3.5, $u^{\Delta t}(t-\Delta t)$ converges to $u$ in $L^{2}\left(\Omega \times \Pi_{T}\right)$. Therefore, in view of the Proposition 3.4 Lipschitz property of $\eta$, and the above discussion we conclude that

$$
\tilde{B}^{\Delta t} \rightarrow \int_{0} \int_{E} \eta(x, u ; z) \tilde{N}(d z, d s) \quad \text { in } L^{2}\left(\Omega \times \Pi_{T}\right) .
$$

Again, note that

$$
\frac{\partial}{\partial t}\left(\tilde{u}^{\Delta t}-\tilde{B}^{\Delta t}\right)(t)=\sum_{k=1}^{N} \frac{\left(u_{k}-u_{k-1}\right)-\left(B_{k}-B_{k-1}\right)}{\Delta t} \mathbf{1}_{[(k-1) \Delta t, k \Delta t)} .
$$


From (3.2), we see that for any $v \in H^{1}\left(\mathbb{R}^{d}\right)$,

$$
\begin{aligned}
& \int_{\mathbb{R}^{d}}\left(\frac{u_{n+1}-u_{n}}{\Delta t}-\frac{1}{\Delta t} \int_{n \Delta t}^{(n+1) \Delta t} \int_{E} \eta\left(x, u_{n} ; z\right) \tilde{N}(d z, d s)\right) v d x \\
& =-\int_{\mathbb{R}^{d}} \nabla \phi\left(u_{n+1}\right) \cdot \nabla v d x-\varepsilon \int_{\mathbb{R}^{d}} \nabla u_{n+1} \cdot \nabla v d x-\int_{\mathbb{R}^{d}} F\left(u_{n+1}\right) \cdot \nabla v d x \\
& \leq\left\{\left\|\nabla \phi\left(u_{n+1}\right)\right\|_{L^{2}\left(\mathbb{R}^{d}\right)}+\varepsilon\left\|\nabla u_{n+1}\right\|_{L^{2}\left(\mathbb{R}^{d}\right)}+c_{f}\left\|u_{n+1}\right\|_{L^{2}\left(\mathbb{R}^{d}\right)}\right\}\|v\|_{H^{1}\left(\mathbb{R}^{d}\right)},
\end{aligned}
$$

and hence

$$
\begin{gathered}
\sup _{v \in H^{1}\left(\mathbb{R}^{d}\right) \backslash\{0\}} \frac{\int_{\mathbb{R}^{d}}\left(\frac{u_{n+1}-u_{n}}{\Delta t}-\frac{1}{\Delta t} \int_{n \Delta t}^{(n+1) \Delta t} \int_{E} \eta\left(x, u_{n} ; z\right) \tilde{N}(d z, d s)\right) v d x}{\|v\|_{H^{1}\left(\mathbb{R}^{d}\right)}} \\
\leq\left\|\nabla \phi\left(u_{n+1}\right)\right\|_{L^{2}\left(\mathbb{R}^{d}\right)}+\varepsilon\left\|\nabla u_{n+1}\right\|_{L^{2}\left(\mathbb{R}^{d}\right)}+c_{f}\left\|u_{n+1}\right\|_{L^{2}\left(\mathbb{R}^{d}\right)}
\end{gathered}
$$

This implies that $\frac{\partial}{\partial t}\left(\tilde{u}^{\Delta t}-\tilde{B}^{\Delta t}\right)(t)$ is a bounded sequence in $L^{2}\left(\Omega \times(0, T) ; H^{-1}\left(\mathbb{R}^{d}\right)\right)$.

To prove the second part of the lemma, we recall that $\tilde{B}^{\Delta t} \rightarrow \int_{0}^{\cdot} \int_{E} \eta(x, u ; z) \tilde{N}(d z, d s)$ and $\tilde{u}^{\Delta t} \rightarrow u$ in $L^{2}\left(\Omega \times \Pi_{T}\right)$. In view of the first part of this lemma, one can conclude that, up to a subsequence

$$
\frac{\partial}{\partial t}\left(\tilde{u}^{\Delta t}-\tilde{B}^{\Delta t}\right) \rightarrow \frac{\partial}{\partial t}\left(u-\int_{0}^{\cdot} \int_{E} \eta(x, u ; z) \tilde{N}(d z, d s)\right) \quad \text { in } L^{2}\left(\Omega \times(0, T) ; H^{-1}\left(\mathbb{R}^{d}\right)\right) .
$$

This completes the proof.

3.1.3. Existence of weak solution. As we have emphasized, our aim is to prove the existence of weak solution for viscous problem. For this, it is required to pass the limit as $\Delta t \rightarrow 0$. To this end, let $\alpha \in L^{2}((0, T))$ and $\beta \in L^{2}(\Omega)$. Then, in view of variational formula (3.9), we obtain

$$
\begin{aligned}
& \int_{\Omega \times(0, T)}\left\langle\frac{\partial}{\partial t}\left(\tilde{u}^{\Delta t}-\tilde{B}^{\Delta t}\right), v\right\rangle \alpha \beta d t d P+\varepsilon \int_{\Omega \times \Pi_{T}} \nabla u^{\Delta t} \cdot \nabla v \alpha \beta d x d t d P \\
& \quad+\int_{\Omega \times \Pi_{T}} \nabla \phi\left(u^{\Delta t}\right) \cdot \nabla v \alpha \beta d x d t d P+\int_{\Omega \times \Pi_{T}} f\left(u^{\Delta t}\right) \cdot \nabla v \alpha \beta d x d t d P=0 .
\end{aligned}
$$

We make use of (3.8), Lemmas 3.5 and 3.6 to pass to the limit as $\Delta t \rightarrow 0$ in the above variational formulation and arrive at

$$
\begin{gathered}
\int_{\Omega \times(0, T)}\left\langle\frac{\partial}{\partial t}\left(u-\int_{0}^{t} \int_{E} \eta(x, u ; z) \tilde{N}(d z, d s)\right), v\right\rangle \alpha \beta d t d P+\varepsilon \int_{\Omega \times \Pi_{T}} \nabla u \cdot \nabla v \alpha \beta d x d t d P \\
+\int_{\Omega \times \Pi_{T}}\{\nabla \phi(u) \cdot \nabla v+f(u) \cdot \nabla v\} \alpha \beta d x d t d P=0 .
\end{gathered}
$$

Since $H^{1}\left(\mathbb{R}^{d}\right)$ is a separable Hilbert space, the above formulation (3.17) yields for almost surely $\omega \in \Omega$,

$$
\left\langle\frac{\partial}{\partial t}\left(u-\int_{0}^{t} \int_{E} \eta(x, u ; z) \tilde{N}(d z, d s)\right), v\right\rangle+\int_{\mathbb{R}^{d}}(\varepsilon \nabla u+\nabla \phi(u)+f(u)) \cdot \nabla v d x=0,
$$

for almost every $t \in[0, T]$.

This proves that $u$ is a weak solution of (3.1). Note that, for every $\phi \in H^{1}\left(\mathbb{R}^{d}\right)$, it is easily seen that

$$
\mathbb{E}\left|\frac{1}{\delta} \int_{0}^{\delta} \int_{\mathbb{R}^{d}}\left(u(s, x)-u_{0}(x)\right) \psi(x) d x d s\right| \leq C \Delta t \quad \text { if } \quad \delta<\Delta t
$$

Therefore,

$$
\limsup _{\delta \downarrow 0} \mathbb{E}\left|\frac{1}{\delta} \int_{0}^{\delta} \int_{\mathbb{R}^{d}}\left(u(s, x)-u_{0}(x)\right) \psi(x) d x d s\right| \leq C \Delta t \quad \text { for very } \quad \delta<\Delta t .
$$


Now, by letting $\Delta t \downarrow 0$, we have

$$
\lim _{\delta \downarrow 0} \frac{1}{\delta} \int_{0}^{\delta} \int_{\mathbb{R}^{d}} u(s, x) \psi(x) d x d s=\int_{\mathbb{R}^{d}} u_{0}(x) \psi(x) d x \quad P \text { - almost surely. }
$$

3.2. A priori bounds for viscous solutions. Note that for fixed $\varepsilon>0$, there exists a weak solution, denoted as $u_{\varepsilon} \in H^{1}\left(\mathbb{R}^{d}\right)$, which satisfies the following variational formulation: $P$ - almost surely in $\Omega$, and almost every $t \in(0, T)$,

$$
\begin{gathered}
\left\langle\frac{\partial}{\partial t}\left[u_{\varepsilon}-\int_{0}^{t} \int_{E} \eta\left(\cdot, u_{\varepsilon}(s, \cdot) ; z\right) \tilde{N}(d z, d s)\right], v\right\rangle+\int_{\mathbb{R}^{d}} \nabla \phi\left(u_{\varepsilon}(t, x)\right) \cdot \nabla v d x \\
+\int_{\mathbb{R}^{d}}\left\{f\left(u_{\varepsilon}(t, x)\right)+\varepsilon \nabla u_{\varepsilon}(t, x)\right\} \cdot \nabla v d x=0,
\end{gathered}
$$

for any $v \in H^{1}\left(\mathbb{R}^{d}\right)$. Let $\beta(u)=u^{2}$. Applying Itô-Lévy formula (cf. Theorem A.1 in the Appendix) to $\beta(u)$, one gets that for any $t>0$

$$
\begin{aligned}
& \int_{\mathbb{R}^{d}} u_{\varepsilon}^{2}(t) d x+2 \int_{0}^{t} \int_{\mathbb{R}^{d}}\left[\varepsilon+\phi^{\prime}\left(u_{\varepsilon}(s)\right)\right]\left|\nabla u_{\varepsilon}\right|^{2} d s+2 \int_{\mathbb{R}^{d}} f\left(u_{\varepsilon}\right) \cdot \nabla u_{\varepsilon} d x \\
& =\int_{\mathbb{R}^{d}} u_{\varepsilon}^{2}(0) d x+2 \int_{0}^{t} \int_{E} \int_{\mathbb{R}^{d}} \int_{0}^{1} \eta\left(x, u_{\varepsilon}(s, x) ; z\right)\left(u_{\varepsilon}+\theta \eta\left(x, u_{\varepsilon} ; z\right)\right) d \theta d x \tilde{N}(d z, d s) \\
& \quad+\int_{0}^{t} \int_{E} \int_{\mathbb{R}^{d}} \eta^{2}\left(x, u_{\varepsilon}(s, x) ; z\right) d x m(d z) d s .
\end{aligned}
$$

Note that $\int_{\mathbb{R}^{d}} f\left(u_{\varepsilon}\right) \cdot \nabla u_{\varepsilon} d x=0$ as $u_{\varepsilon} \in H^{1}\left(\mathbb{R}^{d}\right)$. Taking expectation, we obtain

$$
\begin{gathered}
\mathbb{E}\left[\left\|u_{\varepsilon}(t)\right\|_{2}^{2}\right]+\varepsilon \int_{0}^{t} \mathbb{E}\left[\left\|\nabla u_{\varepsilon}\right\|_{2}^{2}\right] d s+\int_{0}^{t} \mathbb{E}\left[\left\|\nabla G\left(u_{\varepsilon}(s)\right)\right\|_{2}^{2}\right] d s \\
\leq \mathbb{E}\left[\left\|u_{\varepsilon}(0)\right\|_{2}^{2}+C t\|g\|_{L^{2}\left(\mathbb{R}^{d}\right)}^{2}+C \int_{0}^{t} \mathbb{E}\left[\left\|u_{\varepsilon}(s)\right\|_{2}^{2}\right] d s .\right.
\end{gathered}
$$

An application of Gronwall's inequality yields

$$
\sup _{0 \leq t \leq T} \mathbb{E}\left[\left\|u_{\varepsilon}(t)\right\|_{2}^{2}\right]+\varepsilon \int_{0}^{T} \mathbb{E}\left[\left\|\nabla u_{\varepsilon}(s)\right\|_{2}^{2}\right] d s+\int_{0}^{T} \mathbb{E}\left[\left\|\nabla G\left(u_{\varepsilon}(s)\right)\right\|_{2}^{2}\right] d s \leq C .
$$

The achieved results can be summarized into the following theorem.

Theorem 3.7. For any $\varepsilon>0$, there exists a weak solution $u_{\varepsilon}$ to the problem (3.1). Moreover, it satisfies the following estimate:

$$
\sup _{0 \leq t \leq T} \mathbb{E}\left[\left\|u_{\varepsilon}(t)\right\|_{2}^{2}\right]+\varepsilon \int_{0}^{T} \mathbb{E}\left[\left\|\nabla u_{\varepsilon}(s)\right\|_{2}^{2}\right] d s+\int_{0}^{T} \mathbb{E}\left[\left\|\nabla G\left(u_{\varepsilon}(s)\right)\right\|_{2}^{2}\right] d s \leq C,
$$

where $G$ is an associated Kirchoff's function of $\phi$, defined by $G(x)=\int_{0}^{x} \sqrt{\phi^{\prime}(r)} d r$.

Remark 3.8. Let us remark that since any solution to (3.1) is an entropy solution, the solution $u_{\epsilon}$ in unique.

\section{Existence OF ENTROPY SOLUTiOn}

In this section, we will prove the existence of entropy solution. In view of the a priori estimates as in (3.19), we can apply Lemmas 4.2 and 4.3 of [7] (see also [4) and show the existence of Young measure-valued limit process solution $u(t, x, \alpha), \alpha \in(0,1)$ associated to the sequence $\left\{u_{\varepsilon}(t, x)\right\}_{\varepsilon}>0$.

The basic strategy in this case is to apply Young measure technique and adapt Kruzkov's doubling method in the presence of noise for viscous solutions with two different parameters and then send the viscous parameters goes to zero. One needs a version of classical $L^{1}$ contraction principle( for conservation laws) to get the uniqueness of Young measure valued limit and show that Young measure valued limit process is independent of the additional (dummy) variable and hence it will imply the point-wise convergence of viscous solutions. 
4.1. Uniqueness of Young measure valued limit process. To do this, we follow the same line of argument as in [3] for the degenerate parabolic part and [7] for the Lévy noise. For the convenience of the reader, we have chosen to provide detailed proofs of a few crucial technical lemmas and the rest are referred to the appropriate resources. In [4, 7], the authors used the fact that $\Delta u_{\varepsilon} \in L^{2}\left(\Omega \times \Pi_{T}\right)$. Note that, in this case, $u_{\varepsilon} \in H^{1}\left(\mathbb{R}^{d}\right)$. Therefore, we need to regularize $u_{\varepsilon}$ by convolution. Let $\left\{\tau_{\kappa}\right\}$ be a sequence of mollifiers in $\mathbb{R}^{d}$. Since $u_{\varepsilon}$ is a viscous solution to the problem (3.1), as shown in the proof of Theorem A.1 $u_{\varepsilon} * \tau_{\kappa}$ is a solution to the problem

$$
\begin{aligned}
\left(u_{\varepsilon} * \tau_{\kappa}\right)-\int_{0}^{t} \Delta\left(\phi\left(u_{\varepsilon}\right) * \tau_{\kappa}\right) d s= & \int_{0}^{t} \operatorname{div}_{x}\left(f\left(u_{\varepsilon}\right) * \tau_{\kappa}\right) d s+\int_{0}^{t} \int_{E}\left(\eta\left(x, u_{\varepsilon} ; z\right) * \tau_{\kappa}\right) \tilde{N}(d z, d s) \\
& +\int_{0}^{t} \varepsilon \Delta\left(u_{\varepsilon} * \tau_{\kappa}(t, x)\right) d s \quad \text { a.e. } t>0, x \in \mathbb{R}^{d} .
\end{aligned}
$$

Note that, for fixed $\varepsilon>0, \Delta\left(u_{\varepsilon} * \tau_{\kappa}\right) \in L^{2}\left(\Omega \times \Pi_{T}\right)$.

Let $\rho$ and $\varrho$ be the standard nonnegative mollifiers on $\mathbb{R}$ and $\mathbb{R}^{d}$ respectively such that $\operatorname{supp}(\rho) \subset$ $[-1,0]$ and $\operatorname{supp}(\varrho)=\bar{B}_{1}(0)$. We define $\rho_{\delta_{0}}(r)=\frac{1}{\delta_{0}} \rho\left(\frac{r}{\delta_{0}}\right)$ and $\varrho \delta(x)=\frac{1}{\delta^{d}} \varrho\left(\frac{x}{\delta}\right)$, where $\delta$ and $\delta_{0}$ are two positive constants. Given a nonnegative test function $\psi \in C_{c}^{1,2}\left([0, \infty) \times \mathbb{R}^{d}\right)$ and two positive constants $\delta$ and $\delta_{0}$, define

$$
\varphi_{\delta, \delta_{0}}(t, x, s, y)=\rho_{\delta_{0}}(t-s) \varrho_{\delta}(x-y) \psi(s, y) .
$$

Clearly $\rho_{\delta_{0}}(t-s) \neq 0$ only if $s-\delta_{0} \leq t \leq s$ and hence $\varphi_{\delta, \delta_{0}}(t, x ; s, y)=0$ outside $s-\delta_{0} \leq t \leq s$.

Let $u_{\theta}(t, x)$ be a weak solution to the viscous problem (3.1) with parameter $\theta>0$ and initial condition $u_{\theta}(0, x)=v_{0}(x)$. Moreover, let $J$ be the standard symmetric nonnegative mollifier on $\mathbb{R}$ with support in $[-1,1]$ and $J_{l}(r)=\frac{1}{l} J\left(\frac{r}{l}\right)$ for $l>0$. We now simply write down Itô-Lévy formula for $u_{\theta}(t, x)$ against the convex entropy triple $\left(\beta(\cdot-k), F^{\beta}(\cdot, k), \phi^{\beta}(\cdot, k)\right)$ and then multiply by $J_{l}\left(u_{\varepsilon} * \tau_{\kappa}(s, y)-k\right)$ for $k \in \mathbb{R}$ and then integrate with respect to $s, y, k$. Taking expectation to the resulting equations, we have

$$
\begin{aligned}
& \theta \mathbb{E}\left[\int_{\Pi_{T}} \int_{\Pi_{T}} \int_{\mathbb{R}} \beta^{\prime \prime}\left(u_{\theta}(t, x)-k\right)\left|\nabla u_{\theta}(t, x)\right|^{2} \varphi_{\delta, \delta_{0}}(t, x, s, y) J_{l}\left(u_{\varepsilon} * \tau_{\kappa}(s, y)-k\right) d k d x d t d y d s\right] \\
& +\mathbb{E}\left[\int_{\Pi_{T}} \int_{\Pi_{T}} \int_{\mathbb{R}} \beta^{\prime \prime}\left(u_{\theta}(t, x)-k\right)\left|\nabla G\left(u_{\theta}(t, x)\right)\right|^{2} \varphi_{\delta, \delta_{0}}(t, x, s, y) J_{l}\left(u_{\varepsilon} * \tau_{\kappa}(s, y)-k\right) d k d x d t d y d s\right] \\
& \leq \mathbb{E}\left[\int_{\Pi_{T}} \int_{\mathbb{R}^{d}} \int_{\mathbb{R}} \beta\left(v_{0}(x)-k\right) \varphi_{\delta, \delta_{0}}(0, x, s, y) J_{l}\left(u_{\varepsilon} * \tau_{\kappa}(s, y)-k\right) d k d x d y d s\right] \\
& +\mathbb{E}\left[\int_{\Pi_{T}} \int_{\Pi_{T}} \int_{\mathbb{R}} \beta\left(u_{\theta}(t, x)-k\right) \partial_{t} \varphi_{\delta, \delta_{0}}(t, x, s, y) J_{l}\left(u_{\varepsilon} * \tau_{\kappa}(s, y)-k\right) d k d x d t d y d s\right] \\
& +\mathbb{E}\left[\int_{\Pi_{T}} \int_{\mathbb{R}} \int_{0}^{T} \int_{E} \int_{\mathbb{R}^{d}} \int_{0}^{1} \eta\left(x, u_{\theta}(t, x) ; z\right) \beta^{\prime}\left(u_{\theta}(t, x)+\tau \eta\left(x, u_{\theta}(t, x) ; z\right)-k\right)\right. \\
& \left.\times \varphi_{\delta, \delta_{0}}(t, x, s, y) d \tau d x \tilde{N}(d z, d t) J_{l}\left(u_{\varepsilon} * \tau_{\kappa}(s, y)-k\right) d k d y d s\right] \\
& +\mathbb{E}\left[\int_{\Pi_{T}} \int_{0}^{T} \int_{E} \int_{\mathbb{R}^{d}} \int_{\mathbb{R}} \int_{0}^{1}(1-\tau) \eta^{2}\left(x, u_{\theta}(t, x) ; z\right) \beta^{\prime \prime}\left(u_{\theta}(t, x)+\tau \eta\left(x, u_{\theta}(t, x) ; z\right)-k\right)\right. \\
& \left.\times \varphi_{\delta, \delta_{0}}(t, x, s, y) J_{l}\left(u_{\varepsilon} * \tau_{\kappa}(s, y)-k\right) d \tau d k d x m(d z) d t d y d s\right] \\
& -\mathbb{E}\left[\int_{\Pi_{T}} \int_{\Pi_{T}} \int_{\mathbb{R}} F^{\beta}\left(u_{\theta}(t, x), k\right) \nabla_{x} \varrho_{\delta}(x-y) \psi(s, y) \rho_{\delta_{0}}(t-s) J_{l}\left(u_{\varepsilon} * \tau_{\kappa}(s, y)-k\right) d k d x d t d y d s\right] \\
& +\mathbb{E}\left[\int_{\Pi_{T}} \int_{\Pi_{T}} \int_{\mathbb{R}} \phi^{\beta}\left(u_{\theta}(t, x), k\right) \Delta_{x} \varrho_{\delta}(x-y) \psi(s, y) \rho_{\delta_{0}}(t-s) J_{l}\left(u_{\varepsilon} * \tau_{\kappa}(s, y)-k\right) d k d x d t d y d s\right] \\
& -\theta \mathbb{E}\left[\int_{\Pi_{T}} \int_{\Pi_{T}} \int_{\mathbb{R}} \beta^{\prime}\left(u_{\theta}(t, x)-k\right) \nabla_{x} u_{\theta}(t, x) \cdot \nabla_{x} \varphi_{\delta, \delta_{0}}(t, x, s, y) J_{l}\left(u_{\varepsilon} * \tau_{\kappa}(s, y)-k\right) d k d x d t d y d s\right] \\
& \text { i.e., } \quad I_{0,1}+I_{0,2} \leq I_{1}+I_{2}+I_{3}+I_{4}+I_{5}+I_{6}+I_{7} \text {. }
\end{aligned}
$$


We now apply Itô-Lévy formula to (4.1) and obtain

$$
\begin{aligned}
& \varepsilon \mathbb{E}\left[\int_{\Pi_{T}} \int_{\Pi_{T}} \int_{\mathbb{R}} \beta^{\prime \prime}\left(u_{\varepsilon} * \tau_{\kappa}-k\right)\left|\nabla\left(u_{\varepsilon} * \tau_{\kappa}\right)\right|^{2} \varphi_{\delta, \delta_{0}} J_{l}\left(u_{\theta}(t, x)-k\right) d k d x d t d y d s\right] \\
& +\mathbb{E}\left[\int_{\Pi_{T}} \int_{\Pi_{T}} \int_{\mathbb{R}} \beta^{\prime \prime}\left(u_{\varepsilon} * \tau_{\kappa}-k\right) \nabla\left(\phi\left(u_{\varepsilon}\right) * \tau_{\kappa}\right) \cdot \nabla\left(u_{\varepsilon} * \tau_{\kappa}\right) \varphi_{\delta, \delta_{0}} J_{l}\left(u_{\theta}(t, x)-k\right) d k d x d t d y d s\right] \\
& \leq \mathbb{E}\left[\int_{\Pi_{T}} \int_{\mathbb{R}^{d}} \int_{\mathbb{R}} \beta\left(u_{\varepsilon} * \tau_{\kappa}(0, y)-k\right) \varphi_{\delta, \delta_{0}}(t, x, 0, y) J_{l}\left(u_{\theta}(t, x)-k\right) d k d x d y d t\right] \\
& +\mathbb{E}\left[\int_{\Pi_{T}} \int_{\Pi_{T}} \int_{\mathbb{R}} \beta\left(u_{\varepsilon} * \tau_{\kappa}(s, y)-k\right) \partial_{s} \phi_{\delta, \delta_{0}} J_{l}\left(u_{\theta}(t, x)-k\right) d k d y d s d x d t\right] \\
& +\mathbb{E}\left[\int_{\Pi_{T}} \int_{0}^{T} \int_{E} \int_{\mathbb{R}} \int_{\mathbb{R}^{d}} \int_{0}^{1}\left(\eta\left(y, u_{\varepsilon} ; z\right) * \tau_{\kappa}\right) \beta^{\prime}\left(u_{\varepsilon} * \tau_{\kappa}+\theta\left(\eta\left(y, u_{\varepsilon} ; z\right) * \tau_{\kappa}\right)-k\right)\right. \\
& \left.\times \varphi_{\delta, \delta_{0}} J_{l}\left(u_{\theta}(t, x)-k\right) d \theta d y d k \tilde{N}(d z, d s) d x d t\right] \\
& +\mathbb{E}\left[\int_{\Pi_{T}} \int_{0}^{T} \int_{E} \int_{\mathbb{R}} \int_{\mathbb{R}^{d}} \int_{0}^{1}(1-\theta)\left(\eta\left(y, u_{\varepsilon} ; z\right) * \tau_{\kappa}\right)^{2} \beta^{\prime \prime}\left(u_{\varepsilon} * \tau_{\kappa}+\theta\left(\eta\left(y, u_{\varepsilon} ; z\right) * \tau_{\kappa}\right)-k\right)\right. \\
& \left.\times \varphi_{\delta, \delta_{0}} J_{l}\left(u_{\theta}(t, x)-k\right) d \theta d y d k m(d z) d s d x d t\right] \\
& -\mathbb{E}\left[\int_{\Pi_{T}} \int_{\Pi_{T}} \int_{\mathbb{R}} \beta^{\prime}\left(u_{\varepsilon} * \tau_{\kappa}-k\right) \nabla\left(\phi\left(u_{\varepsilon}\right) * \tau_{\kappa}\right) \nabla_{y} \varphi_{\delta, \delta_{0}} J_{l}\left(u_{\theta}(t, x)-k\right) d k d x d t d y d s\right] \\
& -\mathbb{E}\left[\int_{\Pi_{T}} \int_{\Pi_{T}} \int_{\mathbb{R}} \beta^{\prime}\left(u_{\varepsilon} * \tau_{\kappa}-k\right)\left(F\left(u_{\varepsilon}\right) * \tau_{\kappa}\right) \nabla_{y} \varphi_{\delta, \delta_{0}} J_{l}\left(u_{\theta}(t, x)-k\right) d k d x d t d y d s\right] \\
& -\mathbb{E}\left[\int_{\Pi_{T}} \int_{\Pi_{T}} \int_{\mathbb{R}} \beta^{\prime \prime}\left(u_{\varepsilon} * \tau_{\kappa}-k\right)\left(F\left(u_{\varepsilon}\right) * \tau_{\kappa}\right) \nabla_{y}\left(u_{\varepsilon} * \tau_{\kappa}\right) \varphi_{\delta, \delta_{0}} J_{l}\left(u_{\theta}(t, x)-k\right) d k d x d t d y d s\right] \\
& -\varepsilon \mathbb{E}\left[\int_{\Pi_{T}} \int_{\Pi_{T}} \int_{\mathbb{R}} \beta^{\prime}\left(u_{\varepsilon} * \tau_{\kappa}(s, y)-k\right) \nabla_{y}\left(u_{\varepsilon} * \tau_{\kappa}\right) \cdot \nabla_{y} \varphi_{\delta, \delta_{0}} J_{l}\left(u_{\theta}(t, x)-k\right) d k d y d s d x d t\right] \\
& \text { i.e., } \quad J_{0,1}+J_{0,2} \leq J_{1}+J_{2}+J_{3}+J_{4}+J_{5}+J_{6}+J_{7}+J_{8} .
\end{aligned}
$$

We now add (4.3) and (4.4) and look for the passage to the limit in various parameter involved. Note that $I_{0,1}$ and $J_{0,1}$ are both positive terms and they are left hand side of the inequalities (4.3) and (4.4) respectively. Hence we can omit these terms. Let us consider the expressions $I_{0,2}$ and $J_{0,2}$. Recall that

$$
I_{0,2}=\mathbb{E}\left[\int_{\Pi_{T}} \int_{\Pi_{T}} \int_{\mathbb{R}} \beta^{\prime \prime}\left(u_{\theta}(t, x)-k\right)\left|\nabla G\left(u_{\theta}(t, x)\right)\right|^{2} \varphi_{\delta, \delta_{0}} J_{l}\left(u_{\varepsilon} * \tau_{\kappa}(s, y)-k\right) d k d x d t d y d s\right]
$$

and

$$
J_{0,2}=\mathbb{E}\left[\int_{\Pi_{T}} \int_{\Pi_{T}} \int_{\mathbb{R}} \beta^{\prime \prime}\left(u_{\varepsilon} * \tau_{\kappa}-k\right) \nabla\left(\phi\left(u_{\varepsilon}\right) * \tau_{\kappa}\right) \cdot \nabla\left(u_{\varepsilon} * \tau_{\kappa}\right) \varphi_{\delta, \delta_{0}} J_{l}\left(u_{\theta}(t, x)-k\right) d k d x d t d y d s\right] .
$$

By using the properties of Lebesgue points, convolutions, and approximations by mollifications one can able to pass to the limit in $I_{0,2}$ and $J_{0,2}$, and conclude the following lemma.

Lemma 4.1. It holds that

$$
\lim _{l \rightarrow 0} \lim _{\kappa \rightarrow 0} \lim _{\delta_{0} \rightarrow 0} I_{0,2}=\mathbb{E}\left[\int_{\Pi_{T}} \int_{\mathbb{R}^{d}} \beta^{\prime \prime}\left(u_{\theta}(t, x)-u_{\varepsilon}(t, y)\right)\left|\nabla G\left(u_{\theta}(t, x)\right)\right|^{2} \psi(t, y) \varrho_{\delta}(x-y) d x d t d y\right]
$$

and

$$
\begin{gathered}
\lim _{l \rightarrow 0} \lim _{\kappa \rightarrow 0} \lim _{\delta_{0} \rightarrow 0} J_{0,2}=\mathbb{E}\left[\int_{\Pi_{T}} \int_{\mathbb{R}^{d}} \beta^{\prime \prime}\left(u_{\varepsilon}(s, y)-u_{\theta}(s, x)\right) \nabla \phi\left(u_{\varepsilon}(s, y)\right) \cdot \nabla u_{\varepsilon}(s, y)\right. \\
\left.\times \psi(s, y) \varrho_{\delta}(x-y) d x d y d s\right] .
\end{gathered}
$$

Lemma 4.2. It follows that

$\limsup _{\theta \rightarrow 0} \limsup _{\varepsilon \rightarrow 0} \lim _{l \rightarrow 0} \lim _{\kappa \rightarrow 0} \lim _{\delta_{0} \rightarrow 0}\left(I_{0,2}+J_{0,2}\right)$ 


$$
\begin{array}{r}
\geq 2 \mathbb{E}\left[\int_{\Pi_{T}} \int_{\mathbb{R}^{d}} \int_{0}^{1} \int_{0}^{1} \int_{\tilde{u}(t, x, \gamma)}^{u(t, y, \alpha)}\left(\int_{s=\sigma}^{\tilde{u}(t, x, \gamma)} \beta^{\prime \prime}(\sigma-s) \sqrt{\phi^{\prime}(s)} d s\right) \sqrt{\phi^{\prime}(\sigma)} d \sigma\right. \\
\left.\times \operatorname{div}_{y} \nabla_{x}\left[\psi(t, y) \varrho_{\delta}(x-y)\right] d \gamma d \alpha d x d y d t\right] .
\end{array}
$$

Proof. In view of Lemma 4.1, we see that

$$
\begin{aligned}
& \lim _{l \rightarrow 0} \lim _{\kappa \rightarrow 0} \lim _{\delta_{0} \rightarrow 0}\left(I_{0,2}+J_{0,2}\right) \\
= & \mathbb{E}\left[\int_{\Pi_{T}} \int_{\mathbb{R}^{d}} \beta^{\prime \prime}\left(u_{\varepsilon}(t, y)-u_{\theta}(t, x)\right)\left(\left|\nabla_{y} G\left(u_{\varepsilon}(t, y)\right)\right|^{2}+\left|\nabla_{x} G\left(u_{\theta}(t, x)\right)\right|^{2}\right) \psi(t, y) \varrho_{\delta}(x-y) d x d y d t\right] .
\end{aligned}
$$

Let $u(t, y, \alpha)$ and $\tilde{u}(t, x, \gamma)$ be Young measure-valued narrow limit associated to the sequences $\left\{u_{\varepsilon}(t, y)\right\}_{\varepsilon>0}$ and $\left\{u_{\theta}(t, x)\right\}_{\theta>0}$ respectively. With these at hand, one can use a similar argument as in [3, Lemma 3.4] and arrive at the conclusion that (4.5) holds.

Let us consider the terms $\left(I_{1}+J_{1}\right)$ coming from initial conditions. Note that $I_{1}=0$ as supp $\rho_{\delta_{0}} \subset\left[-\delta_{0}, 0\right)$. Under a slight modification of the same line arguments as in [7, we arrive at the following lemma

Lemma 4.3. It holds that

$$
\lim _{\theta \rightarrow 0} \lim _{\varepsilon \rightarrow 0} \lim _{l \rightarrow 0} \lim _{\kappa \rightarrow 0} \lim _{\delta_{0} \rightarrow 0}\left(I_{1}+J_{1}\right)=\mathbb{E}\left[\int_{\mathbb{R}^{d}} \int_{\mathbb{R}^{d}} \beta_{\vartheta}\left(v_{0}(x)-u_{0}(y)\right) \psi(0, y) \varrho_{\delta}(x-y) d x d y\right]
$$

and

$$
\begin{aligned}
\lim _{(\vartheta, \delta) \rightarrow(0,0)} & \mathbb{E}\left[\int_{\mathbb{R}^{d}} \int_{\mathbb{R}^{d}} \beta_{\vartheta}\left(v_{0}(x)-u_{0}(y)\right) \psi(0, y) \varrho_{\delta}(x-y) d x d y\right] \\
& =\mathbb{E}\left[\int_{\mathbb{R}^{d}}\left|v_{0}(x)-u_{0}(x)\right| \psi(0, x) d x\right] .
\end{aligned}
$$

We now turn our attention to $\left(I_{2}+J_{2}\right)$. Note that $\partial_{t} \rho_{\delta_{0}}(t-s)=-\partial_{s} \rho_{\delta_{0}}(t-s)$ and $\beta, J_{l}$ are even functions. A simple calculation gives

$$
\begin{aligned}
I_{2}+J_{2}=\mathbb{E}\left[\int _ { \Pi _ { T } } \int _ { \Pi _ { T } } \int _ { \mathbb { R } } \beta \left(u_{\varepsilon} *\right.\right. & \left.\tau_{\kappa}(s, y)-k\right) \partial_{s} \psi(s, y) \rho_{\delta_{0}}(t-s) \varrho_{\delta}(x-y) \\
& \left.\times J_{l}\left(u_{\theta}(t, x)-k\right) d k d y d s d x d t\right]
\end{aligned}
$$

One can pass to the limit in $\left(I_{2}+J_{2}\right)$ and have the following conclusion.

\section{Lemma 4.4. It holds that}

$$
\begin{aligned}
& \lim _{\theta \rightarrow 0} \lim _{\varepsilon \rightarrow 0} \lim _{l \rightarrow 0} \lim _{\kappa \rightarrow 0} \lim _{\delta_{0} \rightarrow 0}\left(I_{2}+J_{2}\right) \\
= & \mathbb{E}\left[\int_{\Pi_{T}} \int_{\mathbb{R}^{d}} \int_{0}^{1} \int_{0}^{1} \beta(u(s, y, \alpha)-\tilde{u}(s, x, \gamma)) \partial_{s} \psi(s, y) \varrho_{\delta}(x-y) d \gamma d \alpha d y d x d s\right],
\end{aligned}
$$

and

$$
\begin{aligned}
\lim _{(\vartheta, \delta) \rightarrow(0,0)} & \mathbb{E}\left[\int_{\Pi_{T}} \int_{\mathbb{R}^{d}} \int_{0}^{1} \int_{0}^{1} \beta(u(s, y, \alpha)-\tilde{u}(s, x, \gamma)) \partial_{s} \psi(s, y) \varrho_{\delta}(x-y) d \gamma d \alpha d y d x d s\right] \\
& =\mathbb{E}\left[\int_{0}^{T} \int_{\mathbb{R}^{d}} \int_{0}^{1} \int_{0}^{1}|u(s, y, \alpha)-\tilde{u}(s, y, \gamma)| \partial_{s} \psi(s, y) d \gamma d \alpha d y d s\right] .
\end{aligned}
$$

Let us consider $I_{6}$ and $J_{5}$. Regarding this, we have the following lemma

Lemma 4.5. It holds that

$$
\begin{aligned}
& \lim _{\theta \downarrow 0} \lim _{\varepsilon \downarrow 0} \lim _{l \downarrow 0} \lim _{\kappa \downarrow 0} \lim _{\delta_{0} \downarrow 0} I_{6} \\
& =\mathbb{E}\left[\int_{\Pi_{T}} \int_{\mathbb{R}^{d}} \int_{0}^{1} \int_{0}^{1} \phi^{\beta}(\tilde{u}(s, x, \gamma), u(s, y, \alpha)) \Delta_{x} \varrho_{\delta}(x-y) \psi(s, y) d \gamma d \alpha d x d y d s\right]
\end{aligned}
$$


and

$$
\begin{aligned}
& \lim _{\theta \downarrow 0} \lim _{\varepsilon \downarrow 0} \lim _{l \downarrow 0} \lim _{\kappa \downarrow 0} \lim _{\delta_{0} \downarrow 0} J_{5} \\
= & \mathbb{E}\left[\int_{\Pi_{T}} \int_{\mathbb{R}^{d}} \int_{[0,1]^{2}} \phi^{\beta}(u(s, y, \alpha), \tilde{u}(s, x, \gamma)) \Delta_{y}\left[\psi(s, y) \varrho_{\delta}(x-y)\right] d \gamma d \alpha d x d y d s\right] .
\end{aligned}
$$

Proof. Let us consider the passage to the limits in $I_{6}$. To do this, we define,

$$
\begin{aligned}
\mathcal{B}_{1} & =\mathbb{E}\left[\int_{\Pi_{T}} \int_{\Pi_{T}} \int_{\mathbb{R}} \phi^{\beta}\left(u_{\theta}(t, x), k\right) \Delta_{x} \varrho_{\delta}(x-y) \psi(s, y) \rho_{\delta_{0}}(t-s) J_{l}\left(u_{\varepsilon} * \tau_{\kappa}(s, y)-k\right) d k d x d t d y d s\right] \\
& -\mathbb{E}\left[\int_{\Pi_{T}} \int_{\mathbb{R}^{d}} \int_{\mathbb{R}} \phi^{\beta}\left(u_{\theta}(s, x), k\right) \Delta_{x} \varrho_{\delta}(x-y) \psi(s, y) J_{l}\left(u_{\varepsilon} * \tau_{\kappa}(s, y)-k\right) d k d x d y d s\right]
\end{aligned}
$$

Note that, for all $a, b, c \in \mathbb{R}$,

$$
\left|\phi^{\beta}(a, b)-\phi^{\beta}(c, b)\right| \leq C|c-a|
$$

By using (4.6), we have

$$
\begin{aligned}
& \mathcal{B}_{1}= \mathbb{E}\left[\int_{\Pi_{T}} \int_{\Pi_{T}} \int_{\mathbb{R}} \phi^{\beta}\left(u_{\theta}(t, x), u_{\varepsilon} * \tau_{\kappa}(s, y)-k\right) \Delta_{x} \varrho_{\delta}(x-y) \psi(s, y) \rho_{\delta_{0}}(t-s) J_{l}(k) d k d x d t d y d s\right] \\
&- \mathbb{E}\left[\int_{\Pi_{T}} \int_{\mathbb{R}^{d}} \int_{\mathbb{R}} \phi^{\beta}\left(u_{\theta}(s, x), u_{\varepsilon} * \tau_{\kappa}(s, y)-k\right) \Delta_{x} \varrho_{\delta}(x-y) \psi(s, y) J_{l}(k) d k d x d y d s\right] \\
&= \mathbb{E}\left[\int_{\Pi_{T}} \int_{\Pi_{T}} \int_{\mathbb{R}}\left(\phi^{\beta}\left(u_{\theta}(t, x), u_{\varepsilon} * \tau_{\kappa}(s, y)-k\right)-\phi^{\beta}\left(u_{\theta}(s, x), u_{\varepsilon} * \tau_{\kappa}(s, y)-k\right)\right) \Delta_{x} \varrho_{\delta}(x-y)\right. \\
&\left.\times \psi(s, y) \rho_{\delta_{0}}(t-s) J_{l}(k) d k d x d t d y d s\right] \\
&- \mathbb{E}\left[\int_{\Pi_{T}} \int_{\mathbb{R}^{d}} \int_{\mathbb{R}} \phi^{\beta}\left(u_{\theta}(s, x), u_{\varepsilon} * \tau_{\kappa}(s, y)-k\right) \psi(s, y)\left(1-\int_{0}^{T} \rho_{\delta_{0}}(t-s) d t\right)\right. \\
&\text { Then } \left.\quad \times \Delta_{x} \varrho \delta(x-y) J_{l}(k) d k d x d y d s\right] . \\
&\left|\mathcal{B}_{1}\right| \leq C \mathbb{E}\left[\int_{s=\delta_{0}}^{T} \int_{\Pi_{T}} \int_{\mathbb{R}^{d}} \int_{\mathbb{R}}\left|u_{\theta}(t, x)-u_{\theta}(s, x)\right|\left|\Delta_{x} \varrho_{\delta}(x-y)\right| \psi(s, y) \rho_{\delta_{0}}(t-s) J_{l}(k) d k d x d t d y d s\right] \\
& \leq C \mathbb{E}\left[\int_{s=\delta_{0}}^{T} \int_{0}^{T} \int_{K_{\delta}}\left|u_{\theta}(t, x)-u_{\theta}(s, x)\right| \rho_{\delta_{0}}(t-s) d x d t d s\right]+\mathcal{O}\left(\delta_{0}\right) \\
& \leq C \mathbb{E}\left[\int_{r=0}^{1} \int_{0}^{T} \int_{K_{\delta}}\left|u_{\theta}\left(t+\delta_{0} r, x\right)-u_{\theta}(t, x)\right| \rho(-r) d x d t d r\right]+\mathcal{O}\left(\delta_{0}\right)
\end{aligned}
$$

where $K_{\delta} \subset \mathbb{R}^{d}$ is a compact set depending on $\psi$ and $\delta$.

Note that, $\lim _{\delta_{0} \downarrow 0} \int_{0}^{T} \int_{K_{\delta}}\left|u_{\theta}\left(t+\delta_{0} r, x\right)-u_{\theta}(t, x)\right| d x d t \rightarrow 0$ almost surely for all $r \in[0,1]$. Therefore, by dominated convergence theorem, we have

$$
\lim _{\delta_{0} \rightarrow 0} I_{6}=\mathbb{E}\left[\int_{\Pi_{T}} \int_{\mathbb{R}^{d}} \int_{\mathbb{R}} \phi^{\beta}\left(u_{\theta}(s, x), k\right) \Delta_{x} \varrho_{\delta}(x-y) \psi(s, y) J_{l}\left(u_{\varepsilon} * \tau_{\kappa}(s, y)-k\right) d k d x d y d s\right] .
$$

Moreover, one can use the property of convolution to conclude

$$
\lim _{\kappa \rightarrow 0} \lim _{\delta_{0} \rightarrow 0} I_{6}=\mathbb{E}\left[\int_{\Pi_{T}} \int_{\mathbb{R}^{d}} \int_{\mathbb{R}} \phi^{\beta}(v(s, x), k) \Delta_{x} \varrho_{\delta}(x-y) \psi(s, y) J_{l}\left(u_{\varepsilon}(s, y)-k\right) d k d x d y d s\right] .
$$

Passage to the limit as $l \rightarrow 0$ :

$$
\text { Let, } \begin{aligned}
\mathcal{B}_{2}: & =\mathbb{E}\left[\int_{\Pi_{T}} \int_{\mathbb{R}^{d}} \int_{\mathbb{R}} \phi^{\beta}\left(u_{\theta}(s, x), k\right) \Delta_{x} \varrho_{\delta}(x-y) \psi(s, y) J_{l}\left(u_{\varepsilon}(s, y)-k\right) d k d x d y d s\right] \\
& -\mathbb{E}\left[\int_{\Pi_{T}} \int_{\mathbb{R}^{d}} \phi^{\beta}\left(u_{\theta}(s, x), u_{\varepsilon}(s, y)\right) \Delta_{x} \varrho_{\delta}(x-y) \psi(s, y) d x d y d s\right]
\end{aligned}
$$




$$
\begin{gathered}
=\mathbb{E}\left[\int_{\Pi_{T}} \int_{\mathbb{R}^{d}} \int_{\mathbb{R}}\left(\phi^{\beta}\left(u_{\theta}(s, x), k\right)-\phi^{\beta}\left(u_{\theta}(s, x), u_{\varepsilon}(s, y)\right)\right) \Delta_{x} \varrho_{\delta}(x-y) \psi(s, y)\right. \\
\left.\times J_{l}\left(u_{\varepsilon}(s, y)-k\right) d k d x d y d s\right] .
\end{gathered}
$$

Note that for all $a, b, c \in \mathbb{R}$,

$$
\left|\phi^{\beta}(a, b)-\phi^{\beta}(a, c)\right| \leq C(1+|a-b|)|b-c| .
$$

Therefore, by (4.7) we have

$$
\begin{aligned}
\left|\mathcal{B}_{2}\right| \leq C \mathbb{E}\left[\int_{\Pi_{T}} \int_{\mathbb{R}^{d}} \int_{\mathbb{R}}\left(1+\left|u_{\theta}(s, x)-k\right|\right)\left|u_{\varepsilon}(s, y)-k\right|\left|\Delta_{x} \varrho_{\delta}(x-y)\right| \psi(s, y)\right. \\
\left.\times J_{l}\left(u_{\varepsilon}(s, y)-k\right) d k d x d y d s\right] \\
\leq C l\left\{1+\left(\sup _{\theta>0} \sup _{t>0} \mathbb{E}\left[\left\|u_{\theta}(t)\right\|_{2}^{2}\right]\right)^{\frac{1}{2}}+\left(\operatorname{supsup}_{\varepsilon>0} \mathbb{E}\left[\left\|u_{\varepsilon}(t)\right\|_{2}^{2}\right]\right)^{\frac{1}{2}}\right\} \rightarrow 0 \quad \text { as } \quad l \rightarrow 0 .
\end{aligned}
$$

One can justify the passage to the limit as $\varepsilon \rightarrow 0$ and $\theta \rightarrow 0$ in the sense of Young measures as in 4, 7 and conclude

$$
\begin{aligned}
& \lim _{\theta \rightarrow 0} \lim _{\varepsilon \rightarrow 0} \mathbb{E}\left[\int_{\Pi_{T}} \int_{\mathbb{R}^{d}} \phi^{\beta}\left(u_{\theta}(s, x), u_{\varepsilon}(s, y)\right) \Delta_{x} \varrho_{\delta}(x-y) \psi(s, y) d x d y d s\right] \\
= & \mathbb{E}\left[\int_{\Pi_{T}} \int_{\mathbb{R}^{d}} \int_{0}^{1} \int_{0}^{1} \phi^{\beta}(\tilde{u}(s, x, \gamma), u(s, y, \alpha)) \Delta_{x} \varrho_{\delta}(x-y) \psi(s, y) d \gamma d \alpha d x d y d s\right] .
\end{aligned}
$$

This proves the first part of the lemma.

To prove the second part, let us recall that

$$
J_{5}:=-\mathbb{E}\left[\int_{\Pi_{T}} \int_{\Pi_{T}} \int_{\mathbb{R}} \beta^{\prime}\left(u_{\varepsilon} * \tau_{\kappa}-k\right) \nabla\left(\phi\left(u_{\varepsilon}\right) * \tau_{\kappa}\right) \nabla_{y} \varphi_{\delta, \delta_{0}} J_{l}\left(u_{\theta}(t, x)-k\right) d k d x d t d y d s\right]
$$

A classical properties of Lebesgue points and convolution yields

$$
\begin{gathered}
\lim _{\kappa \rightarrow 0} \lim _{\delta_{0} \rightarrow 0} J_{5}=-\mathbb{E}\left[\int_{\Pi_{T}} \int_{\mathbb{R}^{d}} \int_{\mathbb{R}} \beta^{\prime}\left(u_{\varepsilon}(s, y)-k\right) \nabla \phi\left(u_{\varepsilon}(s, y)\right) \cdot \nabla_{y}\left[\psi(s, y) \varrho_{\delta}(x-y)\right]\right. \\
\left.\times J_{l}\left(u_{\theta}(s, x)-k\right) d k d x d y d s\right] .
\end{gathered}
$$

Recall that $\phi^{\beta}(a, b)=\int_{b}^{a} \beta^{\prime}(s-b) \phi^{\prime}(s) d s$. Making use of Green's type formula along with Young measure theory and keeping in mind that $u(s, y, \alpha)$ and $\tilde{u}(s, x, \gamma)$ are Young measure-valued limit processes associated to the sequences $\left\{u_{\varepsilon}(s, y)\right\}_{\varepsilon>0}$ and $\left\{u_{\theta}(s, x)\right\}_{\theta>0}$ respectively, we arrive at the following conclusion

$$
\begin{aligned}
& \lim _{\theta \downarrow 0} \lim _{\varepsilon \downarrow 0} \lim _{l \downarrow 0} \lim _{\kappa \rightarrow 0} \lim _{\delta_{0} \rightarrow 0} J_{5} \\
& =\mathbb{E}\left[\int_{\Pi_{T}} \int_{\mathbb{R}^{d}} \int_{0}^{1} \int_{0}^{1} \phi^{\beta}(u(s, y, \alpha), \tilde{u}(s, x, \gamma)) \Delta_{y}\left[\psi(s, y) \varrho_{\delta}(x-y)\right] d \gamma d \alpha d x d y d s\right] .
\end{aligned}
$$

This completes the proof of the lemma.

Next, we want to pass to the limits in $\left(J_{6}+J_{7}\right)$ and $I_{5}$ respectively. A slight modification of the similar argument as in [3, 4, 7, yields the following lemma:

Lemma 4.6. It holds that

$$
\begin{aligned}
& \lim _{l \rightarrow 0} \lim _{\kappa \rightarrow 0} \lim _{\delta_{0} \rightarrow 0}\left(J_{6}+J_{7}\right) \\
= & -\mathbb{E}\left[\int_{\Pi_{T}} \int_{\mathbb{R}^{d}} F^{\beta}\left(u_{\varepsilon}(s, y), u_{\theta}(s, x)\right) \cdot \nabla_{y}\left[\psi(s, y) \varrho_{\delta}(x-y)\right] d x d y d s\right] \\
\underset{\varepsilon \rightarrow 0}{\longrightarrow} & \mathbb{E}\left[\int_{\Pi_{T}} \int_{\mathbb{R}^{d}} \int_{0}^{1} F^{\beta}\left(u(s, y, \alpha), u_{\theta}(s, x)\right) \cdot \nabla_{y}\left[\psi(s, y) \varrho_{\delta}(x-y)\right] d \alpha d x d y d s\right]
\end{aligned}
$$




$$
\underset{\theta \rightarrow 0}{\longrightarrow}-\mathbb{E}\left[\int_{\Pi_{T}} \int_{\mathbb{R}^{d}} \int_{0}^{1} \int_{0}^{1} F^{\beta}(u(s, y, \alpha), \tilde{u}(s, x, \gamma)) \cdot \nabla_{y}\left[\psi(s, y) \varrho_{\delta}(x-y)\right] d \gamma d \alpha d x d y d s\right]
$$

and

$$
\begin{aligned}
& \lim _{\theta \rightarrow 0} \lim _{\varepsilon \rightarrow 0} \lim _{l \rightarrow 0} \lim _{\kappa \rightarrow 0} \lim _{\delta_{0} \rightarrow 0} I_{5} \\
= & -\mathbb{E}\left[\int_{\Pi_{T}} \int_{\mathbb{R}^{d}} \int_{0}^{1} \int_{0}^{1} F^{\beta}(\tilde{u}(s, x, \gamma), u(s, y, \alpha)) \cdot \nabla_{x}\left[\varrho_{\delta}(x-y) \psi(s, y)\right] d \gamma d \alpha d x d y d s\right] .
\end{aligned}
$$

In view of the above, we now want to pass the limit as $(\vartheta, \delta) \longrightarrow(0,0)$. We use similar line argument as in the proof of the second part of the Lemmas 5.7 and 5.8 in [7] and arrive at the following lemma.

Lemma 4.7. Assume that $\vartheta \rightarrow 0, \delta \rightarrow 0$ and $\frac{\vartheta}{\delta} \rightarrow 0$, then

$$
\begin{aligned}
& \lim _{\vartheta \rightarrow 0} \lim _{\delta \rightarrow 0} \lim _{\frac{\vartheta}{\delta} \rightarrow 0} \lim _{\theta \downarrow 0} \lim _{\varepsilon \downarrow 0} \lim _{l \rightarrow 0} \lim _{\kappa \rightarrow 0} \lim _{\delta_{0} \rightarrow 0}\left[\left(J_{6}+J_{7}\right)+I_{5}\right] \\
& =-\mathbb{E}\left[\int_{\Pi_{T}} \int_{0}^{1} \int_{0}^{1} F(u(s, y, \alpha), \tilde{u}(s, y, \gamma)) \cdot \nabla_{y} \psi(s, y) d \gamma d \alpha d y d s\right] .
\end{aligned}
$$

Let us consider the term $I_{7}+J_{8}$. Regarding this, we have the following

Lemma 4.8. For fixed $\delta>0$ and $\beta$, it holds that

$$
\limsup _{\left(\theta, \varepsilon, l, \kappa, \delta_{0},\right) \rightarrow 0}\left|I_{7}+J_{8}\right|=0
$$

Proof. Note that

$$
\begin{aligned}
\left|J_{8}\right| & \leq \varepsilon\left\|\beta^{\prime}\right\|_{\infty} \mathbb{E}\left[\int_{\Pi_{T}} \int_{\mathbb{R}^{d}}\left|\nabla_{y}\left(u_{\varepsilon} * \tau_{\kappa}(s, y)\right)\right| \mid \nabla_{y}\left[\psi(s, y) \varrho_{\delta}(x-y) \mid d y d x d s\right]\right. \\
& \leq \varepsilon\left\|\beta^{\prime}\right\|_{\infty} \mathbb{E}\left[\int_{|y| \leq K} \int_{0}^{T} \int_{\mathbb{R}^{d}}\left|\nabla_{y}\left(u_{\varepsilon} * \tau_{\kappa}(t, y)\right)\right|\left|\nabla_{y}\left[\psi(t, y) \varrho_{\delta}(x-y)\right]\right| d x d t d y\right]
\end{aligned}
$$

(By Cauchy-Schwartz inequality),

$$
\begin{aligned}
& \leq C(\beta) \varepsilon^{\frac{1}{2}}\left(\mathbb{E}\left[\int_{\Pi_{T}} \varepsilon\left|\nabla_{y}\left(u_{\varepsilon} * \tau_{\kappa}(t, y)\right)\right|^{2} d y d t\right]\right)^{\frac{1}{2}}\left(\mathbb{E}\left[\int_{K} \int_{\Pi_{T}}\left|\nabla_{y}\left[\psi(t, y) \varrho_{\delta}(x-y)\right]\right|^{2} d x d t d y\right]\right)^{\frac{1}{2}} \\
& \leq C(\beta, \psi, \delta) \varepsilon^{\frac{1}{2}}\left(\sup _{\varepsilon>0} \mathbb{E}\left[\left.\left|\varepsilon \int_{0}^{T} \int_{\mathbb{R}^{d}}\right| \nabla_{y} u_{\varepsilon}(t, y)\right|^{2} d y d t \mid\right]\right)^{\frac{1}{2}} \leq C(\beta, \psi, \delta) \varepsilon^{\frac{1}{2}} .
\end{aligned}
$$

Similarly, we have $\left|I_{7}\right| \leq C(\beta, \psi, \delta) \theta^{\frac{1}{2}}$. This completes the proof.

Next we consider the stochastic terms $I_{3}+J_{3}$. To this end, we cite [7] and assert that for two constants $T_{1}, T_{2} \geq 0$ with $T_{1}<T_{2}$,

$$
\mathbb{E}\left[X_{T_{1}} \int_{T_{1}}^{T_{2}} \int_{E} J(t, z) \tilde{N}(d z, d t)\right]=0
$$

where $J$ is a predictable integrand and $X$ is an adapted process.

For each $\beta \in C^{\infty}(\mathbb{R})$ with $\beta^{\prime}, \beta^{\prime \prime} \in C_{b}(\mathbb{R})$ and nonnegative $\varphi \in C_{c}^{\infty}\left(\Pi_{\infty} \times \Pi_{\infty}\right)$, we define

$$
\begin{aligned}
M[\beta, \varphi](s ; y, v):=\int_{0}^{T} \int_{E} \int_{\mathbb{R}^{d}}\left\{\beta \left(u_{\theta}(r, x)+\right.\right. & \left.\left.\eta\left(x, u_{\theta}(r, x) ; z\right)-v\right)-\beta\left(u_{\theta}(r, x)-v\right)\right\} \\
& \times \varphi(r, x, s, y) d x \tilde{N}(d z, d r)
\end{aligned}
$$

where $0 \leq s \leq T,(y, v) \in \mathbb{R}^{d} \times \mathbb{R}$. Furthermore, we extend the process $u_{\varepsilon} * \tau_{\kappa}(\cdot, y)$ for negative time simply by $u_{\varepsilon} * \tau_{\kappa}(s, y)=u_{\varepsilon}(0, \cdot) * \tau_{k}(y)$ if $s<0$. With this convention, it follows from (4.10) that

$$
\mathbb{E}\left[\int_{\mathbb{R}_{v}} \int_{\Pi_{T}} M\left[\beta, \varphi_{\delta, \delta_{0}}\right](s ; y, v) J_{l}\left(u_{\varepsilon}\left(s-\delta_{0}, y\right)-v\right) d y d s d v\right]=0
$$


and hence we have $J_{3}=0$ and

$$
I_{3}=\mathbb{E}\left[\int_{\mathbb{R}} \int_{\Pi_{T}} M\left[\beta, \varphi_{\delta, \delta_{0}}\right](s ; y, v)\left(J_{l}\left(u_{\varepsilon} * \tau_{\kappa}(s, y)-v\right)-J_{l}\left(u_{\varepsilon} * \tau_{\kappa}\left(s-\delta_{0}, y\right)-v\right)\right) d y d s d v\right] .
$$

Our aim is to pass to the limit into the stochastic terms $I_{3}+J_{3}$. For that, we need some estimate regarding $M\left[\beta, \varphi_{\delta, \delta_{0}}\right]$, a proof of which could be found in 7 .

Lemma 4.9. Let $\gamma \in C^{\infty}(\mathbb{R})$ be function such that $\gamma^{\prime} \in C_{c}^{\infty}(\mathbb{R})$ and $p$ be a positive integer of the form $p=2^{k}$ for some $k \in \mathbb{N}$. If $p \geq d+3$ then there exists a constant $C=C\left(\gamma^{\prime}, \psi, \delta\right)$

$$
\sup _{0 \leq s \leq T}\left(\mathbb{E}\left[\left\|M\left[\gamma, \varphi_{\delta, \delta_{0}}\right](s ; \cdot, \cdot)\right\|_{L^{\infty}\left(\mathbb{R}^{d} \times \mathbb{R}\right)}^{2}\right]\right) \leq \frac{C\left(\gamma^{\prime}, \psi, \delta\right)}{\delta_{0}^{\frac{2(p-1)}{p}}} .
$$

and the following identities hold:

$$
\begin{aligned}
& \partial_{v} M[\gamma, \varphi](s ; y, v)=M\left[-\gamma^{\prime}, \varphi\right](s ; y, v) \\
& \partial_{y_{k}} M[\gamma, \varphi](s ; y, v)=M\left[\gamma, \partial_{y_{k}} \varphi\right](s ; y, v) .
\end{aligned}
$$

Lemma 4.10. It holds that

$$
\begin{aligned}
\lim _{l \rightarrow 0} \lim _{\kappa \rightarrow 0} \lim _{\delta_{0} \rightarrow 0} I_{3} & \\
=\mathbb{E}\left[\int_{\Pi_{T}} \int_{\mathbb{R}^{d}} \int_{E}\right. & \left\{\beta\left(u_{\theta}(r, x)+\eta\left(x, u_{\theta}(r, x) ; z\right)-u_{\varepsilon}(r, y)-\eta\left(y, u_{\varepsilon}(r, y) ; z\right)\right)\right. \\
& -\beta\left(u_{\theta}(r, x)-u_{\varepsilon}(r, y)-\eta\left(y, u_{\varepsilon}(r, y) ; z\right)\right)+\beta\left(u_{\theta}(r, x)-u_{\varepsilon}(r, y)\right) \\
& \left.\left.-\beta\left(u_{\theta}(r, x)+\eta\left(x, u_{\theta}(r, x) ; z\right)-u_{\varepsilon}(r, y)\right)\right\} \psi(r, y) \varrho_{\delta}(x-y) m(d z) d x d y d r\right] .
\end{aligned}
$$

Proof. Note that, for all $y \in \mathbb{R}^{d}, u_{\varepsilon} * \tau_{\kappa}(\cdot, y)$ satisfies

$$
\begin{aligned}
d u_{\varepsilon} * \tau_{\kappa}(s, y)= & \operatorname{div}\left(f\left(u_{\varepsilon}\right) * \tau_{\kappa}(s, y)\right) d s+\Delta\left(\phi\left(u_{\varepsilon}\right) * \tau_{\kappa}(s, y)\right) d s \\
& +\varepsilon \Delta u_{\varepsilon} * \tau_{\kappa}(s, y) d s+\int_{E}\left(\eta\left(\cdot, u_{\varepsilon} ; z\right) * \tau_{\kappa}(s, y)\right) \tilde{N}(d z, d s) .
\end{aligned}
$$

Now apply Itô-Lévy formula on $J_{l}\left(u_{\varepsilon} * \tau_{\kappa}(s, y)-v\right)$ to get

$$
\begin{aligned}
& J_{l}\left(u_{\varepsilon} * \tau_{\kappa}(s, y)-v\right)-J_{l}\left(u_{\varepsilon} * \tau_{\kappa}\left(s-\delta_{0}, y\right)-v\right) \\
&= \int_{s-\delta_{0}}^{s} J_{l}^{\prime}\left(u_{\varepsilon} * \tau_{\kappa}(\sigma, y)-v\right)\left(\operatorname{div}\left(f\left(u_{\varepsilon}\right) * \tau_{\kappa}(\sigma, y)\right)+\varepsilon \Delta\left(u_{\varepsilon} * \tau_{\kappa}(\sigma, y)\right)+\Delta\left(\phi\left(u_{\varepsilon}\right) * \tau_{\kappa}(\sigma, y)\right)\right) d \sigma \\
&+\int_{s-\delta_{0}}^{s} \int_{E}\left(J_{l}\left(u_{\varepsilon} * \tau_{\kappa}(\sigma, y)+\left(\eta\left(\cdot, u_{\varepsilon} ; z\right) * \tau_{\kappa}(\sigma, y)\right)-v\right)-J_{l}\left(u_{\varepsilon} * \tau_{\kappa}(\sigma, y)-v\right)\right) \tilde{N}(d z, d \sigma) \\
&+\int_{s-\delta_{0}}^{s} \int_{E} \int_{\lambda=0}^{1}(1-\lambda) J_{l}^{\prime \prime}\left(u_{\varepsilon} * \tau_{\kappa}(\sigma, y)-v+\lambda\left(\eta\left(\cdot, u_{\varepsilon} ; z\right) * \tau_{\kappa}(\sigma, y)\right)\right) \\
&= \times \frac{\partial}{\partial v} \int_{s-\delta_{0}}^{s}\left(\eta\left(\cdot, u_{\varepsilon} ; z\right) * \tau_{\kappa}(\sigma, y)\right)^{2} d \lambda m(d z) d \sigma \\
&\left.+\int_{s-\delta_{0}}^{s} \int_{E}\left(J_{l}\left(u_{\varepsilon} * \tau_{\kappa}(\sigma, y)+\left(\eta\left(\cdot, u_{\varepsilon} ; z\right) * \tau_{\kappa}(\sigma, y)\right)+\varepsilon \Delta\left(u_{\varepsilon} * \tau_{\kappa}(\sigma, y)\right)+\Delta\left(\phi\left(u_{\varepsilon}\right) * \tau_{\kappa}(\sigma, y)\right)\right)-v\right)-J_{l}\left(u_{\varepsilon} * \tau_{\kappa}(\sigma, y)-v\right)\right) \tilde{N}(d z, d \sigma) \\
&+\int_{s-\delta_{0}}^{s} \int_{E} \int_{\lambda=0}^{1}(1-\lambda) J_{l}^{\prime \prime}\left(u_{\varepsilon} * \tau_{\kappa}(\sigma, y)-v+\lambda\left(\eta\left(\cdot, u_{\varepsilon} ; z\right) * \tau_{\kappa}(\sigma, y)\right)\right) \\
& \times\left(\eta\left(\cdot, u_{\varepsilon} ; z\right) * \tau_{\kappa}(\sigma, y)\right)^{2} d \lambda m(d z) d \sigma
\end{aligned}
$$


Therefore, from (4.11) and Lemma 4.9, we have

$$
\begin{aligned}
& I_{3}=-\mathbb{E}\left[\int_{\mathbb{R}} \int_{\Pi_{T}} M\left[\beta^{\prime}, \varphi_{\delta, \delta_{0}}\right](s ; y, v)\left(\int_{s-\delta_{0}}^{s} J_{l}\left(u_{\varepsilon} * \tau_{\kappa}(\sigma, y)-v\right) \operatorname{div}\left(f\left(u_{\varepsilon}\right) * \tau_{\kappa}(\sigma, y)\right) d \sigma\right) d s d y d v\right] \\
&- \mathbb{E}\left[\int_{\mathbb{R}} \int_{\Pi_{T}} M\left[\beta^{\prime}, \varphi_{\delta, \delta_{0}}\right](s ; y, v)\left(\int_{s-\delta_{0}}^{s} J_{l}\left(u_{\varepsilon} * \tau_{\kappa}(\sigma, y)-v\right) \varepsilon \Delta\left(u_{\varepsilon} * \tau_{\kappa}(\sigma, y)\right) d \sigma\right) d s d y d v\right] \\
&- \mathbb{E}\left[\int_{\mathbb{R}} \int_{\Pi_{T}} M\left[\beta^{\prime}, \varphi_{\delta, \delta_{0}}\right](s ; y, v)\left(\int_{s-\delta_{0}}^{s} J_{l}\left(u_{\varepsilon} * \tau_{\kappa}(\sigma, y)-v\right) \Delta\left(\phi\left(u_{\varepsilon}\right) * \tau_{\kappa}(\sigma, y)\right) d \sigma\right) d s d y d v\right] \\
&+ \mathbb{E}\left[\int_{\Pi_{T}} \int_{\mathbb{R}} \int_{s-\delta_{0}}^{s} \int_{\mathbb{R}^{d}} \int_{E}\left(\beta\left(u_{\theta}(r, x)+\eta\left(x, u_{\theta}(r, x) ; z\right)-v\right)-\beta\left(u_{\theta}(r, x)-v\right)\right)\right. \\
& \times\left(J_{l}\left(u_{\varepsilon} * \tau_{\kappa}(r, y)+\eta\left(\cdot, u_{\varepsilon} ; z\right) * \tau_{\kappa}(r, y)-v\right)-J_{l}\left(u_{\varepsilon} * \tau_{\kappa}(r, y)-v\right)\right)\left.\times \rho_{\delta_{0}}(r-s) \psi(s, y) \varrho_{\delta}(x-y) m(d z) d x d r d v d y d s\right] \\
&+ \mathbb{E}\left[\int _ { \mathbb { R } } \int _ { \Pi _ { T } } M [ \beta , \phi _ { \delta , \delta _ { 0 } } ] ( s ; y , v ) \left\{\int_{s-\delta_{0}}^{s} \int_{E} \int_{\lambda=0}^{1} J_{l}^{\prime \prime}\left(u_{\varepsilon} * \tau_{\kappa}(\sigma, y)-v+\lambda\left(\eta\left(\cdot, u_{\varepsilon} ; z\right) * \tau_{\kappa}(\sigma, y)\right)\right)\right.\right. \\
& \equiv A_{1}^{\kappa, l, \varepsilon}\left(\delta, \delta_{0}\right)+A_{2}^{\kappa, l, \varepsilon}\left(\delta, \delta_{0}\right)+A_{3}^{\kappa, l, \varepsilon}\left(\delta, \delta_{0}\right)+B^{\varepsilon, l, \kappa}+A_{4}^{\kappa, l, \varepsilon}\left(\delta, \delta_{0}\right) .
\end{aligned}
$$

Note that, for fixed $\kappa$ and $\varepsilon, \Delta\left(u_{\varepsilon} * \tau_{\kappa}\right) \in L^{2}\left(\Omega \times \Pi_{T}\right)$. One can use Young's inequality for convolution and replace $u_{\varepsilon}$ by $u_{\varepsilon} * \tau_{\kappa}$ to adapt the same line of argument as in [7] and conclude

$$
A_{1}^{\kappa, l, \varepsilon}\left(\delta, \delta_{0}\right) \rightarrow 0, \quad A_{2}^{\kappa, l, \varepsilon}\left(\delta, \delta_{0}\right) \rightarrow 0, \quad A_{3}^{\kappa, l, \varepsilon}\left(\delta, \delta_{0}\right) \rightarrow 0, \quad \text { and } \quad A_{4}^{\kappa, l, \varepsilon}\left(\delta, \delta_{0}\right) \rightarrow 0 \quad \text { as } \delta_{0} \rightarrow 0 .
$$

Again it is routine to pass to the limit for $B^{\varepsilon, l, \kappa}$ and arrive at the conclusion that

$$
\begin{aligned}
\lim _{l \rightarrow 0} \lim _{\kappa \rightarrow 0} \lim _{\delta_{0} \rightarrow 0} B^{\varepsilon, l, \kappa} & \\
=\mathbb{E}\left[\int_{\Pi_{T}} \int_{\mathbb{R}^{d}} \int_{E}\{\right. & \beta\left(u_{\theta}(r, x)+\eta\left(x, u_{\theta}(r, x) ; z\right)-u_{\varepsilon}(r, y)-\eta\left(y, u_{\varepsilon}(r, y) ; z\right)\right) \\
& -\beta\left(u_{\theta}(r, x)-u_{\varepsilon}(r, y)-\eta\left(y, u_{\varepsilon}(r, y) ; z\right)\right)+\beta\left(u_{\theta}(r, x)-u_{\varepsilon}(r, y)\right) \\
& \left.\left.\quad-\beta\left(u_{\theta}(r, x)+\eta\left(x, u_{\theta}(r, x) ; z\right)-u_{\varepsilon}(r, y)\right)\right\} \psi(r, y) \varrho_{\delta}(x-y) m(d z) d x d y d r\right] .
\end{aligned}
$$

This completes the proof.

Let us consider the additional terms $I_{4}+J_{4}$. A similar line arguments as in [3, 4, 7, along with classical properties of convolution yields the following:

\section{Lemma 4.11.}

$$
\begin{array}{r}
\lim _{l \rightarrow 0} \lim _{\kappa \rightarrow 0} \lim _{\delta_{0} \rightarrow 0} J_{4}=\mathbb{E}\left[\int_{\Pi_{T}} \int_{\mathbb{R}^{d}} \int_{E} \int_{\lambda=0}^{1}(1-\lambda) \beta^{\prime \prime}\left(u_{\varepsilon}(s, y)-u_{\theta}(s, x)+\lambda \eta\left(y, u_{\varepsilon}(s, y) ; z\right)\right)\right. \\
\left.\times \eta^{2}\left(y, u_{\varepsilon}(s, y) ; z\right) \psi(s, y) \varrho_{\delta}(x-y) d \lambda m(d z) d x d y d s\right],
\end{array}
$$

and

$$
\begin{aligned}
\lim _{l \rightarrow 0} \lim _{\kappa \rightarrow 0} \lim _{\delta_{0} \rightarrow 0} I_{4}=\mathbb{E}\left[\int_{\Pi_{T}} \int_{\mathbb{R}^{d}} \int_{E} \int_{\lambda=0}^{1}(1-\lambda) \beta^{\prime \prime}\left(u_{\theta}(s, x)-u_{\varepsilon}(s, y)+\lambda \eta\left(x, u_{\theta}(s, x) ; z\right)\right)\right. \\
\left.\times \eta^{2}\left(x, u_{\theta}(s, x) ; z\right) \psi(s, x) \varrho_{\delta}(x-y) d \lambda m(d z) d x d y d s\right] .
\end{aligned}
$$

Now we add these terms (cf. Lemma 4.11) with the terms coming from Lemma 4.10 and have the following lemma.

Lemma 4.12. Assume that $\vartheta \rightarrow 0+, \delta \rightarrow 0+$ and $\vartheta^{-1} \delta^{2} \rightarrow 0+$, then

$$
\limsup _{\vartheta \rightarrow 0+, \delta \rightarrow 0+, \vartheta^{-1} \delta^{2} \rightarrow 0+} \limsup _{\theta, \varepsilon \rightarrow 0}\left[\lim _{l \rightarrow 0} \lim _{\kappa \rightarrow 0} \lim _{\delta_{0} \rightarrow 0}\left(\left(I_{3}+J_{3}\right)+\left(I_{4}+J_{4}\right)\right)\right]=0 \text {. }
$$


Proof. In view of Lemmas 4.10 and 4.11, we see that

$$
\begin{gathered}
\lim _{l \rightarrow 0} \lim _{\kappa \rightarrow 0} \lim _{\delta_{0} \rightarrow 0}\left(\left(I_{3}+J_{3}\right)+\left(I_{4}+J_{4}\right)\right) \\
=\mathbb{E}\left[\int _ { \Pi _ { T } } \int _ { \mathbb { R } ^ { d } } \left(\int _ { E } \left\{\beta\left(u_{\theta}(t, x)-u_{\varepsilon}(t, y)+\eta\left(x, u_{\theta}(t, x) ; z\right)-\eta\left(y, u_{\varepsilon}(t, y) ; z\right)\right)\right.\right.\right. \\
-\left(\eta\left(x, u_{\theta}(t, x) ; z\right)-\eta\left(y, u_{\varepsilon}(t, y) ; z\right)\right) \beta^{\prime}\left(u_{\theta}(t, x)-u_{\varepsilon}(t, y)\right) \\
\left.\left.\left.-\beta\left(u_{\theta}(t, x)-u_{\varepsilon}(t, y)\right)\right\} m(d z)\right) \psi(t, y) \varrho_{\delta}(x-y) d x d y d t\right] \\
=\mathbb{E}\left[\int_{\Pi_{T}} \int_{\mathbb{R}^{d}}\left(\int_{E} \int_{\tau=0}^{1} b^{2}(1-\tau) \beta^{\prime \prime}(a+\tau b) d \tau m(d z)\right) \psi(t, y) \varrho_{\delta}(x-y) d x d y d t\right],
\end{gathered}
$$

where $a=u_{\theta}(t, x)-u_{\varepsilon}(t, y)$ and $b=\eta\left(x, u_{\theta}(t, x) ; z\right)-\eta\left(y, u_{\varepsilon}(t, y) ; z\right)$. By using the similar argument as one used in the proof of [7, Lemma 5.11], we arrive at

$$
\lim _{l \rightarrow 0} \lim _{\kappa \rightarrow 0} \lim _{\delta_{0} \rightarrow 0}\left(\left(I_{3}+J_{3}\right)+\left(I_{4}+J_{4}\right)\right) \leq C_{1}\left(\vartheta+\vartheta^{-1} \delta^{2}\right) T,
$$

where the constant $C_{1}$ depends only on $\psi$ and is in particular independent of $\varepsilon$. We now let $\vartheta \rightarrow 0$, $\delta \rightarrow 0$ and $\vartheta^{-1} \delta^{2} \rightarrow 0$, yielding

$$
\limsup _{\vartheta \rightarrow 0, \delta \rightarrow 0, \vartheta^{-1}} \limsup _{\delta^{2} \rightarrow 0}\left[\lim _{\ell \rightarrow \varepsilon \rightarrow 0} \lim _{\kappa \rightarrow 0} \lim _{\delta_{0} \rightarrow 0}\left(\left(I_{3}+J_{3}\right)+\left(I_{4}+J_{4}\right)\right)\right] \leq 0 .
$$

This concludes the proof as $\lim _{l \rightarrow 0} \lim _{\kappa \rightarrow 0} \lim _{\delta_{0} \rightarrow 0}\left(\left(I_{3}+J_{3}\right)+\left(I_{4}+J_{4}\right)\right) \geq 0$, thanks to (4.14).

We now turn our attention back to the terms which are coming from the Lemmas 4.2 and 4.5 To this end, define

$$
\begin{aligned}
\mathcal{H}:= & -2 \mathbb{E}\left[\int_{\Pi_{T}} \int_{\mathbb{R}^{d}} \int_{0}^{1} \int_{0}^{1} \int_{\tilde{u}(s, x, \gamma)}^{u(s, y, \alpha)}\left(\int_{r=\sigma}^{\tilde{u}(s, x, \gamma)} \beta^{\prime \prime}(\sigma-r) \sqrt{\phi^{\prime}(r)} d r\right) \sqrt{\phi^{\prime}(\sigma)} d \sigma\right. \\
& \left.\times \operatorname{div}_{y} \nabla_{x}\left[\psi(s, y) \varrho_{\delta}(x-y)\right] d \gamma d \alpha d x d y d s\right] \\
+ & \mathbb{E}\left[\int_{\Pi_{T}} \int_{\mathbb{R}^{d}} \int_{0}^{1} \int_{0}^{1} \phi^{\beta}(\tilde{u}(s, x, \gamma), u(s, y, \alpha)) \Delta_{x} \varrho_{\delta}(x-y) \psi(s, y) d \gamma d \alpha d x d y d s\right] \\
& +\mathbb{E}\left[\int_{\Pi_{T}} \int_{\mathbb{R}^{d}} \int_{0}^{1} \int_{0}^{1} \phi^{\beta}(u(s, y, \alpha), \tilde{u}(s, x, \gamma)) \Delta_{y}\left[\psi(s, y) \varrho_{\delta}(x-y)\right] d \gamma d \alpha d x d y d s\right] \\
= & \mathbb{E}\left[\int _ { \Pi _ { T } } \int _ { \mathbb { R } ^ { d } } \int _ { 0 } ^ { 1 } \int _ { 0 } ^ { 1 } \left\{2 I_{\beta}(\tilde{u}(s, x, \gamma), u(s, y, \alpha))+\phi^{\beta}(\tilde{u}(s, x, \gamma), u(s, y, \alpha))\right.\right. \\
+ & \mathbb{E}\left[\int_{\Pi_{T}} \int_{\mathbb{R}^{d}} \int_{0}^{1} \int_{0}^{1}\left(2 I_{\beta}(u(s, y, \alpha), \tilde{u}(s, x, \gamma))+2 \phi^{\beta}(\tilde{u}(s, x, \gamma), u(s, y, \alpha))\right)\right. \\
& +\mathbb{E}^{\beta}\left[\int_{\Pi_{T}} \int_{\mathbb{R}^{d}} \int_{0}^{1} \int_{0}^{1} \phi^{\beta}(u(s, y, \alpha), \tilde{u}(s, x, \gamma)) \Delta_{y} \psi(s, y) \varrho_{\delta}(x-y) d \gamma d \alpha d x d y d s\right] \\
\equiv & +\mathcal{H}_{2}+\mathcal{H}_{3},
\end{aligned}
$$

where

$$
I_{\beta}(a, b)=\int_{a}^{b} \int_{\mu}^{a} \beta^{\prime \prime}(\mu-\sigma) \sqrt{\phi^{\prime}(\sigma)} d \sigma \sqrt{\phi^{\prime}(\mu)} d \mu \quad \text { for any } \quad a, b \in \mathbb{R} .
$$

Our aim is to pass the limit in $\mathcal{H}$ as $(\vartheta, \delta) \rightarrow(0,0)$. For this, we need some a priori estimates on $I_{\beta}(a, b)$. Here we state the required lemma whose proof could be found in [3]. 
Lemma 4.13. The following holds:

1.) $I_{\beta}(a, b)=I_{\beta}(b, a) \quad$ and $\quad I_{\beta}(a, b)=-\frac{1}{2} \int_{a}^{b} \int_{a}^{b} \beta^{\prime \prime}(\sigma-\mu) \sqrt{\phi^{\prime}(\mu)} \sqrt{\phi^{\prime}(\sigma)} d \mu d \sigma$.

2.) $2 I_{\beta}(a, b)+\phi^{\beta}(a, b)+\phi^{\beta}(b, a)=\frac{1}{2} \int_{a}^{b} \int_{a}^{b} \beta^{\prime \prime}(\mu-\sigma)\left[\sqrt{\phi^{\prime}(\mu)}-\sqrt{\phi^{\prime}(\sigma)}\right]^{2} d \mu d \sigma$.

Moreover, if $\sqrt{\phi^{\prime}}$ has a modulus of continuity $\omega_{\phi}$, then

$$
2 I_{\beta}(a, b)+\phi^{\beta}(a, b)+\phi^{\beta}(b, a) \leq C|b-a|\left|\omega_{\phi}(|\vartheta|)\right|^{2}
$$

and

$$
2 I_{\beta}(a, b)+\phi^{\beta}(b, a) \leq C|b-a|\left|\omega_{\phi}(|\vartheta|)\right|^{2}+C \min \{2 \vartheta,|b-a|\} .
$$

Let us back to the expression $\mathcal{H}$. Regarding this, we have following:

\section{Lemma 4.14 .}

$$
\lim _{(\vartheta, \delta) \rightarrow(0,0)} \mathcal{H}=\mathbb{E}\left[\int_{\Pi_{T}} \int_{0}^{1} \int_{0}^{1} \mid \phi\left(u(s, y, \alpha)-\phi(\tilde{u}(s, y, \gamma)) \mid \Delta_{y} \psi(s, y) d \gamma d \alpha d y d s\right] .\right.
$$

Proof. Let $\omega_{\phi}$ be a modulus of continuity of $\sqrt{\phi^{\prime}}$. Then, thanks to Lemma 4.13, we obtain

$$
\begin{aligned}
& \left|\mathcal{H}_{1}\right| \leq C \mathbb{E}\left[\int_{\Pi_{T}} \int_{\mathbb{R}^{d}} \int_{0}^{1} \int_{0}^{1}\left|\omega_{\phi}(|\vartheta|)\right|^{2}|u(s, y, \alpha)-\tilde{u}(s, x, \gamma)| \psi(s, y)\left|\Delta_{y} \varrho_{\delta}(x-y)\right| d \gamma d \alpha d x d y d s\right] \\
& \leq C(\psi) \frac{\left|\omega_{\phi}(|\vartheta|)\right|^{2}}{\delta^{2}}
\end{aligned}
$$

and

$$
\begin{aligned}
& \quad\left|\mathcal{H}_{2}\right| \\
& \leq C \mathbb{E}\left[\int_{\Pi_{T}} \int_{\mathbb{R}^{d}} \int_{0}^{1} \int_{0}^{1}\left|\omega_{\phi}(|\vartheta|)\right|^{2}|u(s, y, \alpha)-\tilde{u}(s, x, \gamma)|\left|\nabla_{y} \psi(s, y)\right|\left|\nabla_{y} \varrho_{\delta}(x-y)\right| d \gamma d \alpha d x d y d s\right] \\
& \quad+\mathbb{E}\left[\int_{\Pi_{T}} \int_{\mathbb{R}^{d}} C \vartheta\left|\nabla_{y} \psi(s, y)\right|\left|\nabla_{y} \varrho_{\delta}(x-y)\right| d x d y d s\right] \\
& \leq C(\psi) \frac{\left|\omega_{\phi}(|\vartheta|)\right|^{2}}{\delta}+C \frac{\vartheta}{\delta} .
\end{aligned}
$$

Hence, we have

$$
\left|\mathcal{H}_{1}\right|+\left|\mathcal{H}_{2}\right| \leq C(\psi) \frac{\left|\omega_{\phi}(|\vartheta|)\right|^{2}}{\delta^{2}}+C(\psi) \frac{\left|\omega_{\phi}(|\vartheta|)\right|^{2}}{\delta}+C \frac{\vartheta}{\delta} .
$$

Put $\delta=\vartheta^{\frac{2}{3}}$. Then, by our assumption (A.1) we see that

$$
\lim _{(\vartheta, \delta) \rightarrow(0,0)}\left(\mathcal{H}_{1}+\mathcal{H}_{2}\right)=0 .
$$

To prove the lemma, it is now required to show

$$
\lim _{(\vartheta, \delta) \rightarrow(0,0)} \mathcal{H}_{3}=\mathbb{E}\left[\int_{\Pi_{T}} \int_{0}^{1} \int_{0}^{1}|\phi(u(s, y, \alpha))-\phi(u(s, y, \gamma))| \Delta_{y} \psi(s, y) d \gamma d \alpha d y d s\right]
$$

and this follows easily as $(a, b) \longmapsto|\phi(a)-\phi(b)|$ is Lipschitz continuous and

$$
\left|\phi^{\beta_{\vartheta}}(a, b)-\right| \phi(a)-\phi(b)|| \leq C \vartheta \quad \text { for any } \quad a, b \in \mathbb{R} .
$$

All of the above results can now be combined into the following proposition. 
Proposition 4.15. Let $\tilde{u}(t, x, \gamma)$ and $u(t, x, \alpha)$ be the predictable process with initial data $v(0, x)$ and $u(0, x)$ respectively which have been extracted out of Young measure valued sub-sequential limit of the sequence $\left\{u_{\theta}(t, x)\right\}_{\theta>0}$ and $\left\{u_{\varepsilon}(t, x)\right\}_{\varepsilon>0}$ respectively. Then, for any non-negative $H^{1}\left([0, \infty) \times \mathbb{R}^{d}\right)$ function $\psi(t, x)$ with compact support, the following inequality holds

$$
\begin{aligned}
0 \leq & \mathbb{E}\left[\int_{\mathbb{R}^{d}}\left|v_{0}(x)-u_{0}(x)\right| \psi(0, x) d x\right]+\mathbb{E}\left[\int_{\Pi_{T}} \int_{0}^{1} \int_{0}^{1}|\tilde{u}(t, x, \gamma)-u(t, x, \alpha)| \partial_{t} \psi(t, x) d \gamma d \alpha d x d t\right] \\
- & \mathbb{E}\left[\int_{\Pi_{T}} \int_{0}^{1} \int_{0}^{1} F(u(t, x, \alpha), \tilde{u}(t, x, \gamma)) \cdot \nabla_{x} \psi(t, x) d \gamma d \alpha d x d t\right] \\
& -\mathbb{E}\left[\int_{\Pi_{T}} \nabla\left(\int_{0}^{1} \int_{0}^{1}|\phi(u(t, x, \alpha))-\phi(\tilde{u}(t, x, \gamma))| d \gamma d \alpha\right) \cdot \nabla_{x} \psi(t, x) d x d t\right] .
\end{aligned}
$$

Proof. First we add (4.3) and (4.4) and then pass to the limit $\lim _{\varepsilon \downarrow 0} \lim _{l \rightarrow 0} \lim _{\kappa \rightarrow 0} \lim _{\delta_{0} \downarrow 0}$. Invoking the Lemmas 4.2, 4.3, 4.4, 4.5, 4.6, 4.8 and 4.12, we put $\delta=\vartheta^{\frac{2}{3}}$ in the resulting expression and then let $\vartheta \rightarrow 0$ with the second parts of Lemmas 4.3. 4.4. Keeping in mind the Lemmas 4.7, 4.12 and 4.14. we conclude that (4.16) holds for any nonnegative test function $\psi \in C_{c}^{2}\left([0, \infty) \times \mathbb{R}^{d}\right)$. It now follows by routine approximation argument that (4.16) holds for any $\psi$ with compact support such that $\psi \in H^{1}\left([0, \infty) \times \mathbb{R}^{d}\right)$. This completes the proof.

Remark 4.16. Note that the same Proposition holds without assuming the existence of a modulus of continuity for $\phi^{\prime}$ if $\eta$ is not a function of $x$. Indeed, it is possible to pass to the limit first on the parameter $\vartheta$, then $\delta$, in Lemmas 4.3 4.4, 4.7 and 4.12. Thus, if one assumes that $\eta$ is not a function of the space variable $x$, it is also possible to pass to the limit first on the parameter $\vartheta$, then $\delta$, in Lemma 4.12 since one would have

$$
\lim _{l \rightarrow 0} \lim _{\kappa \rightarrow 0} \lim _{\delta_{0} \rightarrow 0}\left(\left(I_{3}+J_{3}\right)+\left(I_{4}+J_{4}\right)\right) \leq C_{1} \vartheta T
$$

in its proof. Then, the result holds following [3, first situation p.523].

Our aim is to show the uniqueness of $u(t, x, \alpha)$ and $\tilde{u}(t, x, \gamma)$. To do this, here we follow the ideas of [1, 3], and define for each $n \in \mathbb{N}$,

$$
\phi_{n}(x)= \begin{cases}1, & \text { if }|x| \leq n \\ \frac{n^{a}}{|x|^{a}} & \text { if }|x|>n\end{cases}
$$

where $a=\frac{d}{2}+\tilde{\varepsilon}$ in which $\tilde{\varepsilon}>0$ could be chosen such a way that $\phi_{n} \in L^{2}\left(\mathbb{R}^{d}\right)$. Also, for each $h>0$ and fixed $t \geq 0$, we define

$$
\psi_{h}^{t}(s)=\left\{\begin{array}{l}
1, \quad \text { if } s \leq t \\
1-\frac{s-t}{h}, \quad \text { if } t \leq s \leq t+h \\
0, \quad \text { if } s>t+h
\end{array}\right.
$$

A straightforward calculation revels that,

$$
\begin{aligned}
& \nabla \phi_{n}(x)=-a \frac{\phi_{n}(x)}{|x|} \frac{x}{|x|} \mathbf{1}_{|x|>n} \in L^{2}\left(\mathbb{R}^{d}\right)^{d} \\
& \Delta \phi_{n}(x)=a(2+2 \tilde{\varepsilon}-a) \frac{\phi_{n}(x)}{|x|^{2}} \in L^{2}(\{|x|>n\}) .
\end{aligned}
$$

Clearly (4.16) holds with $\psi(s, x)=\phi_{n}(x) \psi_{h}^{t}(s)$. Thus, for a.e $t \geq 0$, we obtain

$$
\begin{aligned}
& \frac{1}{h} \int_{t}^{t+h} \mathbb{E}\left[\int_{\mathbb{R}^{d}} \int_{0}^{1} \int_{0}^{1}|u(s, x, \alpha)-\tilde{u}(s, x, \gamma)| \phi_{n}(x) d \gamma d \alpha d x\right] d s \\
& \leq \mathbb{E}\left[\int_{0}^{T} \int_{\{|x|>n\}} \int_{0}^{1} \int_{0}^{1}|\phi(u(s, x, \alpha))-\phi(\tilde{u}(s, x, \gamma))| \Delta \phi_{n}(x) \psi_{h}^{t}(s) d \gamma d \alpha d x d s\right] \\
& \quad-\mathbb{E}\left[\int_{0}^{T} \int_{\{|x|>n\}} \int_{0}^{1} \int_{0}^{1} F(u(s, x, \alpha), \tilde{u}(s, x, \gamma)) \cdot \nabla \phi_{n}(x) \psi_{h}^{t}(s) d \gamma d \alpha d x d s\right]
\end{aligned}
$$




$$
\begin{aligned}
-\mathbb{E} & {\left[\int_{0}^{T} \psi_{h}^{t}(s)\left(\int_{\partial\{|x|>n\}} \int_{0}^{1} \int_{0}^{1}|\phi(u(s, x, \alpha))-\phi(\tilde{u}(s, x, \gamma))| \nabla \phi_{n}(x) \cdot \tilde{n} d \gamma d \alpha d x\right) d s\right] } \\
& +\mathbb{E}\left[\int_{\mathbb{R}^{d}}\left|v_{0}(x)-u_{0}(x)\right| \phi_{n}(x) d x\right] .
\end{aligned}
$$

Since $\nabla \phi_{n}(x) \cdot \tilde{n}=\frac{a}{n}>0$ on the set $\partial\{|x|>n\}$, we have, from (4.17)

$$
\begin{aligned}
& \frac{1}{h} \int_{t}^{t+h} \mathbb{E}\left[\int_{\mathbb{R}^{d}} \int_{0}^{1} \int_{0}^{1}|u(s, x, \alpha)-\tilde{u}(s, x, \gamma)| \phi_{n}(x) d \gamma d \alpha d x\right] d s \\
& \leq \mathbb{E}\left[\int_{0}^{T} \int_{\{|x|>n\}} \int_{0}^{1} \int_{0}^{1} a(2+2 \tilde{\varepsilon}-a) \frac{\phi_{n}(x)}{|x|^{2}}|\phi(u(s, x, \alpha))-\phi(\tilde{u}(s, x, \gamma))| \psi_{h}^{t}(s) d \gamma d \alpha d x d s\right] \\
& \quad+\mathbb{E}\left[\int_{0}^{T} \int_{\{|x|>n\}} \int_{0}^{1} \int_{0}^{1} a \frac{\phi_{n}(x)}{|x|} F(u(s, x, \alpha), \tilde{u}(s, x, \gamma)) \cdot \frac{x}{|x|} \psi_{h}^{t}(s) d \gamma d \alpha d x d s\right] \\
& \quad+\mathbb{E}\left[\int_{\mathbb{R}^{d}}\left|v_{0}(x)-u_{0}(x)\right| \phi_{n}(x) d x\right] .
\end{aligned}
$$

Note that $|F(a, b)| \leq c_{f}|a-b|$ for any $a, b \in \mathbb{R}$. Since $\phi$ is Lipschitz continuous function and $n \geq 1$, inequality (4.18) gives

$$
\begin{aligned}
& \frac{1}{h} \int_{t}^{t+h} \mathbb{E}\left[\int_{\mathbb{R}^{d}} \int_{0}^{1} \int_{0}^{1}|u(s, x, \alpha)-\tilde{u}(s, x, \gamma)| \phi_{n}(x) d \gamma d \alpha d x\right] d s \\
\leq & C \mathbb{E}\left[\int_{\Pi_{T}} \int_{[0,1]^{2}}|u(s, x, \alpha)-\tilde{u}(s, x, \gamma)| \phi_{n}(x) \psi_{h}^{t}(s) d \gamma d \alpha d x d s\right]+\mathbb{E}\left[\int_{\mathbb{R}^{d}}\left|v_{0}(x)-u_{0}(x)\right| \phi_{n}(x) d x\right] .
\end{aligned}
$$

Taking limit as $h \rightarrow 0$, and then using a weaker version of Gronwall's inequality, we obtain, for a.e. $t>0$,

$$
\mathbb{E}\left[\int_{\mathbb{R}^{d}} \int_{0}^{1} \int_{0}^{1}|u(t, x, \alpha)-\tilde{u}(t, x, \gamma)| \phi_{n}(x) d \gamma d \alpha d x\right] \leq e^{C T} \mathbb{E}\left[\int_{\mathbb{R}^{d}}\left|v_{0}(x)-u_{0}(x)\right| \phi_{n}(x) d x\right] .
$$

Thus, if we assume that $v_{0}(x)=u_{0}(x)$, then we arrive at the conclusion

$$
\mathbb{E}\left[\int_{\mathbb{R}^{d}} \int_{0}^{1} \int_{0}^{1}|u(t, x, \alpha)-\tilde{u}(t, x, \gamma)| \phi_{n}(x) d \gamma d \alpha d x\right]=0,
$$

which says that for almost all $\omega \in \Omega$, a.e. $(t, x) \in(0, T] \times \mathbb{R}^{d}$ and a.e. $(\alpha, \gamma) \in[0,1]^{2}, u(t, x, \alpha)=$ $\tilde{u}(t, x, \gamma)$. On the other hand, we conclude that the whole sequence of viscous approximation converges weakly in $L^{2}\left(\Omega \times \Pi_{T}\right)$. Since the limit process is independent of the additional (dummy) variable, the viscous approximation converges strongly in $L^{p}\left(\Omega \times(0, T) ; L^{p}(\Theta)\right)$ for any $p<2$ and any bounded open set $\Theta \subset \mathbb{R}^{d}$.

4.2. Existence of entropy solution. In this subsection, using strong convergence of viscous solutions and a priori bounds (3.19) we establish the existence of entropy solution to the underlying problem (1.1).

Fix a nonnegative test function $\psi \in C_{c}^{\infty}\left([0, \infty) \times \mathbb{R}^{d}\right), B \in \mathcal{F}_{T}$ and convex entropy flux triple $(\beta, \zeta, \nu)$. Now apply Itô-Lévy formula (3.1) and conclude

$$
\begin{aligned}
& \mathbb{E}\left[\mathbf{1}_{B} \int_{\Pi_{T}} \beta^{\prime \prime}\left(u_{\varepsilon}(t, x)\right)\left|\nabla G\left(u_{\varepsilon}(t, x)\right)\right|^{2} \psi(t, x) d x d t\right] \\
\leq & \mathbb{E}\left[\mathbf{1}_{B} \int_{\mathbb{R}^{d}} \beta\left(u_{\varepsilon}(0, x)\right) \psi(0, x) d x\right]-\varepsilon \mathbb{E}\left[\mathbf{1}_{B} \int_{\Pi_{T}} \beta^{\prime}\left(u_{\varepsilon}(t, x)\right) \nabla u_{\varepsilon}(t, x) \cdot \nabla \psi(t, x) d x d t\right] \\
+ & \mathbb{E}\left[\mathbf{1}_{B} \int_{\Pi_{T}}\left(\beta\left(u_{\varepsilon}(t, x)\right) \partial_{t} \psi(t, x)+\nu\left(u_{\varepsilon}(t, x)\right) \Delta \psi(t, x)-\nabla \psi(t, x) \cdot \zeta\left(u_{\varepsilon}(t, x)\right)\right) d x d t\right] \\
+ & \mathbb{E}\left[\mathbf{1}_{B} \int_{0}^{T} \int_{E} \int_{\mathbb{R}^{d}} \int_{0}^{1} \eta\left(x, u_{\varepsilon}(t, x) ; z\right) \beta^{\prime}\left(u_{\varepsilon}(t, x)+\theta \eta\left(x, u_{\varepsilon}(t, x) ; z\right)\right) \psi(t, x) d \theta d x \tilde{N}(d z, d t)\right] \\
+ & \mathbb{E}\left[\mathbf{1}_{B} \int_{0}^{T} \int_{E} \int_{\mathbb{R}^{d}} \int_{0}^{1}(1-\theta) \eta^{2}\left(x, u_{\varepsilon}(t, x) ; z\right) \beta^{\prime \prime}\left(u_{\varepsilon}(t, x)+\theta \eta\left(x, u_{\varepsilon}(t, x) ; z\right)\right)\right.
\end{aligned}
$$




$$
\times \psi(t, x) d \theta d x m(d z) d t]
$$

Let the predictable process $u(t, x)$ be the pointwise limit of $u_{\varepsilon}(t, x)$ for a.e. $(t, x) \in(0, T) \times \mathbb{R}^{d}$ almost surely. One can now pass to the limit in (4.20) (cf. same argument as in [7]) except the first term. The pointwise limit of $u_{\varepsilon}(t, x)$ is not enough to pass the limit in the first term of the inequality because $u_{\varepsilon}$ is in a gradient term. For this, we proceed as follows: fix $v \in L^{2}\left(\Omega \times \Pi_{T}\right)$. Define

$$
f_{\varepsilon}=\sqrt{\beta^{\prime \prime}\left(u_{\varepsilon}(t, x)\right) \psi(t, x) \mathbf{1}_{B}}, \quad \text { and } \quad g_{\varepsilon}=\nabla G\left(u_{\varepsilon}(t, x)\right) .
$$

Note that, $f_{\varepsilon}$ is uniformly bounded and $g_{\varepsilon} \rightarrow g=\nabla G(u(t, x))$ in $L^{2}\left(\Omega \times \Pi_{T}\right)$. Also, $f_{\varepsilon}$ converges to $f$ pointwise (up to a subsequence) where $f=\sqrt{\beta^{\prime \prime}(u(t, x)) \psi(t, x) \mathbf{1}_{B}}$. Since $\left|f_{\varepsilon} v\right| \leq$ $\sqrt{\left\|\beta^{\prime \prime}\right\|_{\infty} \psi(t, x)}|v(t, x)|$ and right hand side is $L^{2}$ integrable, one can apply dominated convergence theorem to conclude

$$
f_{\varepsilon} v \longrightarrow f v \quad \text { in } L^{2}\left(\Omega \times \Pi_{T}\right) .
$$

Moreover, we have $f_{\varepsilon} g_{\varepsilon} \rightarrow f g$ in $L^{2}\left(\Omega \times \Pi_{T}\right)$ and therefore, by Fatou's lemma for weak convergence,

$$
\begin{aligned}
& \mathbb{E}\left[\mathbf{1}_{B} \int_{\Pi_{T}} \beta^{\prime \prime}(u(t, x))|\nabla G(u(t, x))|^{2} \psi(t, x) d x d t\right] \\
\leq & \liminf _{\varepsilon \downarrow 0} \mathbb{E}\left[\mathbf{1}_{B} \int_{\Pi_{T}} \beta^{\prime \prime}\left(u_{\varepsilon}(t, x)\right)\left|\nabla G\left(u_{\varepsilon}(t, x)\right)\right|^{2} \psi(t, x) d x d t\right] .
\end{aligned}
$$

Thus, we can pass to the limit in (4.20) as $\varepsilon \rightarrow 0$ and arrive at following inequality.

$$
\begin{aligned}
& \mathbb{E}\left[\mathbf{1}_{B} \int_{\Pi_{T}} \beta^{\prime \prime}(u(t, x))|\nabla G(u(t, x))|^{2} \psi(t, x) d x d t\right]-\mathbb{E}\left[\mathbf{1}_{B} \int_{\mathbb{R}^{d}} \beta\left(u_{0}(x)\right) \psi(0, x) d x\right] \\
& \leq \mathbb{E}\left[\mathbf{1}_{B} \int_{\Pi_{T}}\left(\beta(u(t, x)) \partial_{t} \psi(t, x)+\nu(u(t, x)) \Delta \psi(t, x)-\nabla \psi(t, x) \cdot \zeta\left(u_{\varepsilon}(t, x)\right)\right) d x d t\right] \\
&+ \mathbb{E}\left[\mathbf{1}_{B} \int_{0}^{T} \int_{E} \int_{\mathbb{R}^{d}} \int_{0}^{1} \eta(x, u(t, x) ; z) \beta^{\prime}(u(t, x)+\theta \eta(x, u(t, x) ; z)) \psi(t, x) d \theta d x \tilde{N}(d z, d t)\right] \\
&+ \mathbb{E}\left[\mathbf{1}_{B} \int_{0}^{T} \int_{E} \int_{\mathbb{R}^{d}} \int_{0}^{1}(1-\theta) \eta^{2}(x, u(t, x) ; z) \beta^{\prime \prime}(u(t, x)+\theta \eta(x, u(t, x) ; z))\right. \\
&\times \psi(t, x) d \theta d x m(d z) d t] .
\end{aligned}
$$

We are now in a position to prove the existence of entropy solution for the underlying problem (1.1).

Proof of the Theorem 2.3. The uniform moment estimate (3.19) together with a general version of Fatou's lemma gives

$$
\sup _{0 \leq t \leq T} \mathbb{E}\left[\|u(t, \cdot)\|_{2}^{2}\right]<\infty \quad \text { and } \quad\|\nabla G(u)\|_{L^{2}\left(\Omega \times \Pi_{T}\right)}^{2}<\infty .
$$

For any $0 \leq \psi \in C_{c}^{\infty}\left([0, \infty) \times \mathbb{R}^{d}\right)$ and given convex entropy flux triple $(\beta, \zeta, \nu)$, Eq. (4.21) holds for every $B \in \mathcal{F}_{T}$. Hence, the following inequality

$$
\begin{aligned}
& \int_{\mathbb{R}^{d}} \beta\left(u_{0}(x)\right) \psi(0, x) d x+\int_{\Pi_{T}} \beta(u(t, x)) \partial_{t} \psi(t, x) d x d t \\
& +\int_{\Pi_{T}} \nu(u(t, x)) \Delta \psi(t, x) d x d t-\int_{\Pi_{T}} \nabla \psi(t, x) \cdot \zeta(u(t, x)) d x d t \\
& +\int_{0}^{T} \int_{E} \int_{\mathbb{R}^{d}} \int_{0}^{1} \eta(x, u(t, x) ; z) \beta^{\prime}(u(t, x)+\theta \eta(x, u(t, x) ; z)) \psi(t, x) d \theta d x \tilde{N}(d z, d t) \\
& +\int_{E} \int_{\Pi_{T}} \int_{0}^{1}(1-\theta) \eta^{2}(x, u(t, x) ; z) \beta^{\prime \prime}(u(t, x)+\theta \eta(x, u(t, x) ; z)) \psi(t, x) d \theta d x d t m(d z)
\end{aligned}
$$




$$
\geq \int_{\Pi_{T}} \beta^{\prime \prime}(u(t, x))|\nabla G(u(t, x))|^{2} \psi(t, x) d x d t
$$

holds $P$-almost surely. This shows that $u(t, x)$ is an entropy solution of (1.1) in the sense of Definition 2.2. This completes the proof.

We now close this section with a sketch of the justification of our claim in Remark 2.5. To see this, let $h_{\delta}$ denote a smooth even convex approximation of |. $\left.\right|^{p}$ define for positive $x$ by:

$h_{\delta}$ vanishes at 0 and uniquely recovered from its second order derivative defined as $h_{\delta}^{\prime \prime}(x)=x^{p-2}$ is $x \in\left[0, \frac{1}{\delta}\right]$ and $\frac{1}{\delta^{p-2}}$ if $x>\frac{1}{\delta}$.

It holds that , $0 \leq h_{\delta}(x) \nearrow h(x)=K_{p}|x|^{p}$ and there exists $C_{p}$ such that $0 \leq h_{\delta}^{\prime \prime}(x) \leq C_{p} h(x)$. Furthermore, it is easily seen that $h_{\delta}^{\prime \prime}(x+y) \leq \tilde{C}_{p}\left(h_{\delta}^{\prime \prime}(x)+h_{\delta}^{\prime \prime}(y)\right)$.

Note that the weak Itô-Lévy formula in Theorem A.1 makes sense for $\beta=h_{\delta}$, as $h_{\delta}^{\prime \prime}$ is bounded. This enables us write, for almost every $t>0$,

$$
\begin{aligned}
& E \int_{\mathbb{R}^{d}} h_{\delta}\left(u_{\epsilon}\right) d x-E \int_{\mathbb{R}^{d}} h_{\delta}\left(u_{0}\right) d x+E \int_{0}^{t} \int_{\mathbb{R}^{d}}\left(\phi^{\prime}\left(u_{\epsilon}\right)+\epsilon\right) h_{\delta}^{\prime \prime}\left(u_{\epsilon}\right)\left|\nabla u_{\epsilon}\right|^{2} d x d t \\
& =E \int_{0}^{t} \int_{E} \int_{\mathbb{R}^{d}}\left(h_{\delta}\left(u_{\epsilon}+\eta\left(x, u_{\epsilon} ; z\right)\right)-h_{\delta}\left(u_{\epsilon}\right)-\eta\left(x, u_{\epsilon} ; z\right) h_{\delta}^{\prime}\left(u_{\epsilon}\right)\right) d x m(d z) d t . \\
& =E \int_{0}^{t} \int_{E} \int_{\mathbb{R}^{d}} \int_{0}^{1}(1-\theta)\left(\eta\left(x, u_{\epsilon} ; z\right)\right)^{2} h_{\delta}^{\prime \prime}\left(u_{\epsilon}+\theta \eta\left(x, u_{\epsilon} ; z\right)\right) d \theta d x m(d z) d s .
\end{aligned}
$$

We can now use the properties of $h_{\delta}$ and the assumptions on $\eta$ to arrive at

$$
\begin{aligned}
E \int_{\mathbb{R}^{d}} h_{\delta}\left(u_{\epsilon}\right) d x & \leq E \int_{\mathbb{R}^{d}} h_{\delta}\left(u_{0}\right) d x+C_{\eta} E \int_{0}^{t} \int_{\mathbb{R}^{d}}\left(1+u_{\epsilon}^{2}\right) h_{\delta}^{\prime \prime}\left(u_{\epsilon}\right) d s \\
& \leq E \int_{\mathbb{R}^{d}}\left|u_{0}\right|^{p} d x+K_{\eta} E \int_{0}^{t} \int_{\mathbb{R}^{d}} h_{\delta}\left(u_{\epsilon}\right) d s
\end{aligned}
$$

and, by a weak Gronwall inequality, $E \int_{\mathbb{R}^{d}} h_{\delta}\left(u_{\epsilon}\right) d x \leq e^{K_{\eta} t} E \int_{\mathbb{R}^{d}}\left|u_{0}\right|^{p} d x$ for all almost all $t$. This implies $E \int_{\mathbb{R}^{d}}\left|u_{\epsilon}\right|^{p} d x \leq e^{C_{\eta} t} E \int_{\mathbb{R}^{d}}\left|u_{0}\right|^{p} d x$ by monotone convergence theorem. The solution $u$ will inherit the same property by Fatou's lemma.

If $u_{0}$ is bounded and $\eta(x, u ; z)=0$ for $|u| \geq M, M$ been given, then, consider non-negative regular convex function $x \mapsto h(x)=\left[(x+K)^{-}\right]^{2}+\left[(x-K)^{+}\right]^{2}$ where $K=\max \left(M+M_{1},\left\|u_{0}\right\|_{\infty}\right)$. Since $h\left(u_{0}\right)=0$ and $h$ vanishes where $\eta$ is active, the Itô formula Yields $E \int_{\mathbb{R}^{d}}\left|h\left(u_{\epsilon}\right)\right| d x=0$ and $u_{\epsilon}$ is uniformly bounded by $K$. Again, the solution $u$ will inherit the same property by passing to the limit.

\section{UNIQUENESS OF ENTROPY SOLUTION}

To prove the uniqueness of entropy solution, we compare any entropy solution to the viscous solution via Kruzkov's doubling variables method and then pass to the limit as viscous parameter goes to zero. We have already shown that limit of the viscous solutions serve for existence of entropy solution for the underlying problem. Now let $v(t, x)$ be any entropy solution and $u_{\varepsilon}(t, x)$ be viscous solution for the problem (3.1). Then one can use exactly the same argument as in Section 4, and end up with the following equality

$$
\mathbb{E}\left[\int_{\mathbb{R}^{d}} \int_{0}^{1}|u(t, x, \alpha)-v(t, x)| \phi_{n}(x) d \alpha d x\right]=0 .
$$

This implies that, for almost every $t \in[0, \infty), v(t, x)=u(t, x, \alpha)$ for almost every $x \in \mathbb{R}^{d},(\omega, \alpha) \in$ $\Omega \times(0,1)$. In other words, this proves the uniqueness for entropy solutions. 


\section{ApPendix A. WEAK ITÔ-LÉvy FORMUla}

Let $u$ be a $H^{1}\left(\mathbb{R}^{d}\right)$-valued $\mathcal{F}_{t}$-predictable process and it is a weak solution to the SPDE

$$
\begin{gathered}
d u(t, x)-\Delta \phi(u(t, x)) d t=\operatorname{div}_{x} f(u(t, x)) d t+\int_{E} \eta(x, u(t, x) ; z) \tilde{N}(d z, d t) \\
+\varepsilon \Delta u(t, x) d t, \quad t>0, x \in \mathbb{R}^{d} .
\end{gathered}
$$

In addition, in view of (3.8), we further assume that $u \in L^{2}\left((0, T) \times \Omega ; H^{1}\left(\mathbb{R}^{d}\right)\right)$. Moreover, $u$ satisfies the initial condition $u_{0} \in L^{2}\left(\mathbb{R}^{d}\right)$ in the following sense: $P$-almost surely

$$
\lim _{h \rightarrow 0} \frac{1}{h} \int_{0}^{h} \int_{\mathbb{R}^{d}} u(t, x) \phi(x) d x=\int_{\mathbb{R}^{d}} u_{0}(x) \phi(x) d x .
$$

for every $\phi \in C_{c}^{\infty}\left(\mathbb{R}^{d}\right)$. We have the following weak version of Itô-Lévy formula for $u(t, \cdot)$.

Theorem A.1. Let the assumptions $(\mathbf{A} .1)(\mathbf{A} .5)$ hold and $u(t, \cdot)$ be a $H^{1}\left(\mathbb{R}^{d}\right)$-valued weak solution of (A.1), as described in subsection 3.1.3, which satisfies (A.2). Then for every entropy triplet $(\beta, \zeta, \nu)$ and $\psi \in C_{c}^{1,2}\left([0, \infty) \times \mathbb{R}^{d}\right)$, it holds $P$-almost surely that

$$
\begin{aligned}
& \int_{\mathbb{R}^{d}} \beta(u(T, x)) \psi(T, x) d x-\int_{\mathbb{R}^{d}} \beta(u(0, x)) \psi(0, x) d x \\
= & \int_{\Pi_{T}} \beta(u(t, x)) \partial_{t} \psi(t, x) d x d t-\int_{\Pi_{T}} \nabla \psi(t, x) \cdot \zeta(u(t, x)) d x d t \\
+ & \int_{\Pi_{T}} \int_{E} \int_{0}^{1} \eta(x, u(t, x) ; z) \beta^{\prime}(u(t, x)+\theta \eta(x, u(t, x) ; z)) \psi(t, x) d \theta \tilde{N}(d z, d t) d x \\
+ & \int_{\Pi_{T}} \int_{E} \int_{0}^{1}(1-\theta) \eta^{2}(x, u(t, x) ; z) \beta^{\prime \prime}(u(t, x)+\theta \eta(x, u(t, x) ; z)) \psi(t, x) d \theta m(d z) d x d t \\
- & \int_{\Pi_{T}}\left(\varepsilon \nabla \psi(t, x) \cdot \nabla_{x} \beta(u(t, x))+\varepsilon \beta^{\prime \prime}(u(t, x))\left|\nabla_{x} u(t, x)\right|^{2} \psi(t, x)\right) d x d t \\
- & \int_{\Pi_{T}} \phi^{\prime}(u(t, x)) \beta^{\prime \prime}(u(t, x))|\nabla u(t, x)|^{2} \psi(t, x) d x d t+\int_{\Pi_{T}} \nu(u(t, x)) \Delta \psi(t, x) d x d t
\end{aligned}
$$

for almost every $T>0$.

Proof. Let $\left\{\tau_{k}\right\}$ be a standard sequence of mollifiers on $\mathbb{R}^{d}$. Then for every $\rho(\cdot) \in C_{c}^{1}((0, T))$ we have

$$
\begin{aligned}
-\int_{0}^{T} u(s, \cdot) * \tau_{k} \rho^{\prime}(s) d s= & \int_{0}^{T} \rho(s) \Delta\left(\phi(u(s, \cdot)) * \tau_{k}\right) d s+\int_{0}^{T} \rho(s) \operatorname{div}_{x}\left(f(u) * \tau_{\kappa}\right) d s \\
& +\int_{0}^{T} \int_{E} \rho(s)\left(\eta(x, u, z) * \tau_{k}\right) \tilde{N}(d z, d s)+\epsilon \int_{0}^{T} \Delta\left(u * \tau_{k}(s, x)\right) \rho(s) d s
\end{aligned}
$$

holds $P$-almost surely. For every $n \in \mathbb{N}$, define

$$
\rho_{n}^{t}(s)=\left\{\begin{array}{l}
n s \text { if } 0 \leq s \leq \frac{1}{n} \\
1, \quad \text { if } \frac{1}{n} \leq s<t \\
1-n(s-t), \quad \text { if } t+\frac{1}{n}>s \geq t \\
0, \quad \text { elsewhere. }
\end{array}\right.
$$

It follows by standard approximation argument that (A.3) is still valid if we replace $\rho(\cdot)$ by $\rho_{n}^{t}(\cdot)$. Afterwards, we invoke right continuity of stochastic integral and standard facts related to Lebesgue points of Banach space valued functions to pass to the limit $n \rightarrow \infty$ and conclude for almost all $t>0$

$$
u * \tau_{k}(t, \cdot)-u_{0} * \rho_{k}=\int_{0}^{t} \Delta\left(\phi(u(s, \cdot)) * \tau_{k}\right) d s+\int_{0}^{t} \operatorname{div}_{x}\left(f(u) * \tau_{\kappa}\right) d s
$$




$$
+\int_{0}^{t} \int_{E}\left(\eta(x, u, z) * \tau_{k}\right) \tilde{N}(d z, d s)+\epsilon \int_{0}^{t} \Delta\left(u * \tau_{k}(s, x)\right) d s
$$

$P$-holds almost surely. In the above, we have used that the weak solution satisfies the initial condition in the sense of (A.2). Let $\beta$ be the entropy function mentioned in the statement and $\psi$ be the test function specified. Now we apply Itô-Lévy chain rule to $\beta\left(u * \tau_{k}(t, \cdot)\right)$ to have, for almost every $t>0$,

$$
\begin{aligned}
\beta\left(u * \tau_{k}(t, \cdot)\right) & =\beta\left(u_{0} * \rho_{k}\right)+\int_{0}^{t} \beta^{\prime}\left(u * \tau_{k}(t, \cdot)\right) \Delta\left(\phi(u(s, \cdot)) * \tau_{k}\right) d s \\
& +\int_{0}^{t} \beta^{\prime}\left(u * \tau_{k}(t, \cdot)\right) \operatorname{div}_{x}\left(f(u) * \tau_{\kappa}\right) d s+\epsilon \int_{0}^{t} \beta^{\prime}\left(u * \tau_{k}(t, \cdot)\right) \Delta\left(u * \tau_{k}(s, x)\right) d s \\
& +\int_{0}^{t} \int_{E}\left(\beta\left(u * \tau_{k}+\eta(x, u, z) * \tau_{k}\right)-\beta(u * \tau)\right) \tilde{N}(d z, d s) \\
& +\int_{0}^{t} \int_{E}\left(\beta\left(u * \tau_{k}+\eta(x, u, z) * \tau_{k}\right)-\beta(u * \tau)-\eta(x, u, z) * \tau_{k} \beta^{\prime}\left(u * \tau_{k}\right)\right) m(d z) d t
\end{aligned}
$$

P-almost surely. We now apply Itó-Lévy product rule on $\beta\left(u * \tau_{k}\right) \psi(t, x)$ and integrate with respect to $x$ to obtain for almost every $T>0$,

$$
\begin{aligned}
& \int_{\mathbb{R}^{d}} \beta\left(u * \tau_{k}(T, x)\right) \psi(T, x) d x \\
& =\int_{\mathbb{R}^{d}} \beta\left(u_{0} * \rho_{k}\right) \psi(0, x) d x+\int_{0}^{T} \int_{\mathbb{R}^{d}} \beta\left(u * \tau_{k}\right) \partial_{s} \psi(s, x) d x d s \\
& +\int_{0}^{T} \int_{\mathbb{R}^{d}} \beta^{\prime}\left(u * \tau_{k}(s, \cdot)\right) \Delta\left(\phi(u(s, \cdot)) * \tau_{k}\right) \psi(s, x) d x d s \\
& +\int_{0}^{T} \int_{\mathbb{R}^{d}} \beta^{\prime}\left(u * \tau_{k}(s, \cdot)\right) \operatorname{div}_{x}\left(f(u) * \tau_{\kappa}\right) \psi(s, x) d x d s \\
& +\epsilon \int_{0}^{T} \int_{R^{d}} \psi(s, x) \beta^{\prime}\left(u * \tau_{k}(s, \cdot)\right) \Delta\left(u * \tau_{k}(s, x)\right) d x d s \\
& +\int_{0}^{T} \int_{\mathbb{R}^{d}} \int_{E} \psi(s, x)\left(\beta\left(u * \tau_{k}+\eta(x, u, z) * \tau_{k}\right)-\beta(u * \tau)\right) d x \tilde{N}(d z, d s) \\
& +\int_{0}^{T} \int_{\mathbb{R}^{d}} \int_{E} \psi(s, x)\left(\beta\left(u * \tau_{k}+\eta(x, u, z) * \tau_{k}\right)-\beta(u * \tau)-\eta(x, u, z) * \tau_{k} \beta^{\prime}\left(u * \tau_{k}\right)\right) m(d z) d x d s
\end{aligned}
$$

almost surely. Note that $u * \tau_{k}(T, \cdot) \rightarrow u(T, \cdot)$ and $u_{0} * \tau_{k} \rightarrow u_{0}$ in $L^{2}\left(\Omega \times \mathbb{R}^{d}\right)$ as $k \rightarrow 0$. Therefore by Lipschitz continuity of $\beta$, we have $\int_{\mathbb{R}^{d}} \beta\left(u * \tau_{k}(T, x)\right) \psi(T, x) d x \rightarrow \int_{\mathbb{R}^{d}} \beta(u(T, x)) \psi(T, x) d x$ and $\int_{\mathbb{R}^{d}} \beta\left(u_{0} * \rho_{k}\right) \psi(0, x) d x \rightarrow \int_{\mathbb{R}^{d}} \beta\left(u_{0}\right) \psi(0, x) d x$ in $L^{2}(\Omega)$. By a similar reasoning, $\int_{0}^{T} \int_{\mathbb{R}^{d}} \beta(u *$ $\left.\tau_{k}\right) \partial_{s} \psi(s, x) d x d s \rightarrow \int_{0}^{T} \int_{\mathbb{R}^{d}} \beta(u) \partial_{s} \psi(s, x) d x d s$ as $k \rightarrow 0$.

Furthermore, note that

$$
\begin{array}{r}
\int_{0}^{T} \int_{\mathbb{R}^{d}} \beta^{\prime}\left(u * \tau_{k}(t, \cdot)\right) \Delta\left(\phi(u(s, \cdot)) * \tau_{k}\right) \psi(s, x) d s d x \\
\left.=-\int_{0}^{T} \int_{\mathbb{R}^{d}} \nabla_{x}\left(\psi(t, x) \beta^{\prime}\left(u * \tau_{k}(t, x)\right)\right) \cdot\left(\nabla \phi(u()) * \tau_{k}\right)(s, x)\right) d x d s
\end{array}
$$

and $\nabla u, \nabla \phi(u) \in L^{2}\left(0, T ; L^{2}\left(\Omega \times \mathbb{R}^{d}\right)\right)$.

Therefore, $\nabla_{x}\left(\psi(t, x) \beta^{\prime}\left(u * \tau_{k}(t, x)\right)\right) \rightarrow \nabla_{x}\left(\psi(t, x) \beta^{\prime}(u(t, x))\right)$ and $\nabla \phi(u) * \tau_{k} \rightarrow \nabla \phi(u)$ in $L^{2}\left(0, T ; L^{2}\left(\Omega \times \mathbb{R}^{d}\right)\right)$ as $k \rightarrow 0$. Therefore, $\int_{0}^{T} \int_{\mathbb{R}^{d}} \beta^{\prime}\left(u * \tau_{k}(t, \cdot)\right) \Delta\left(\phi(u(s, \cdot)) * \tau_{k}\right) \psi(s, x) d s d x \rightarrow$ 
$-\int_{0}^{T} \int_{\mathbb{R}^{d}} \nabla_{x}\left(\psi(t, x) \beta^{\prime}(u(t, x))\right) \cdot(\nabla \phi(u(s, x)) d x d s$

in $L^{1}(\Omega)$ as $k \rightarrow 0$. By the same reasoning,

$$
\int_{\Pi_{T}} \psi(s, x) \beta^{\prime}\left(u * \tau_{k}(s, x)\right) \Delta\left(u * \tau_{k}(s, x)\right) d x d s \rightarrow-\int_{\Pi_{T}} \nabla_{x}\left(\psi(s, x) \beta^{\prime}(u(s, x))\right) \cdot \nabla(u(s, x)) d x d s .
$$

in $L^{1}(\Omega)$ as $k \rightarrow 0$.

Also, it may be recalled that $\operatorname{div}_{x} f(u) \in L^{2}\left(0, T ; L^{2}\left(\Omega \times \mathbb{R}^{d}\right)\right)$ and $\beta\left(u * \tau_{k}\right) \rightarrow \beta(u)$ in $L^{2}\left(0, T ; L^{2}\left(\Omega \times \mathbb{R}^{d}\right)\right)$ as $\kappa \rightarrow 0$. Therefore,

$$
\int_{0}^{T} \int_{\mathbb{R}^{d}} \beta^{\prime}\left(u * \tau_{k}(t, \cdot)\right) \operatorname{div}_{x}\left(f(u) * \tau_{\kappa}\right) \psi(s, x) d x d s \rightarrow \int_{0}^{T} \int_{\mathbb{R}^{d}} \beta^{\prime}(u) \operatorname{div}_{x}(f(u)) \psi(s, x) d x d s
$$

as $k \rightarrow 0$ in $L^{1}(\Omega)$. To this end, we denote

$$
\begin{aligned}
I_{k}(s, z) & =\int_{\mathbb{R}^{d}} \psi(s, x)\left(\beta\left(u * \tau_{k}+\eta(x, u, z) * \tau_{k}\right)-\beta(u * \tau)\right) d x, \\
I(s, z) & =\int_{\mathbb{R}^{d}} \psi(s, x)(\beta(u+\eta(x, u, z))-\beta(u * \tau)) d x .
\end{aligned}
$$

It follows from straightforward computation that $\int_{0}^{T} \int_{E}\left|I_{k}(s, z)-I(s, z)\right|^{2} m(d z) d s \rightarrow 0$ as $k \rightarrow 0$. Therefore, we can invoke Itô-Lévy isometry and pass to the limit $k \rightarrow 0$, in the martingale term in (A.6). This competes the validation of passage to the limit as $k \rightarrow 0$ in every term of (A.6). The assertion is now concluded by simply letting $k \rightarrow 0$ in (A.6) and rearranging the terms.

\section{REFERENCES}

[1] B. Andreianov, M. Maliki. A note on uniqueness of entropy solutions to degenerate parabolic equations in $\mathbb{R}^{n}$. NoDEA Nonlinear Differential Equations Appl. 17(1) (2010) 109-118.

[2] E. J. Balder. Lectures on Young measure theory and its applications in economics. Rend. Istit. Mat.Univ. Trieste, 31 Suppl. 1:1-69, 2000

[3] C. Bauzet, G. Vallet and P. Wittbold. A degenerate parabolic-hyperbolic Cauchy problem with a stochastic force. J. Hyperbolic Differ. Equ., 12 (2015), no. 3, 501-533.

[4] C. Bauzet, G. Vallet and P. Wittbold. The Cauchy problem for conservation law with a multiplicative stochastic perturbation. J. Hyperbolic Differ. Equ. 9 (2012), no.4, 661-709.

[5] M. Bendahmane and K. H. Karlsen. Renormalized entropy solutions for quasilinear anisotropic degenerate parabolic equations. SIAM J. Math. Anal. 36(2): 405-422, 2004.

[6] I. H. Biswas and A. K. Majee. Stochastic conservation laws: weak-in-time formulation and strong entropy condition. Journal of Functional Analysis 267 (2014) 2199-2252.

[7] I. H. Biswas, K. H. Karlsen and A. K. Majee. Stochastic balance laws driven by Lévy noise. J. Hyperbolic Differ. Equ., 12 (2015), no. 3, 581-654.

[8] I. H. Biswas, U. Koley and A. K. Majee. Continuous dependence estimate for conservation laws with Lévy noise. J. Differential Equations 259 (2015), no. 9, 4683-4706.

[9] H. Brezis. Oprateurs maximaux monotones et semi-groupes de contractions dans les espaces de Hilbert. North-Holland, New York, American Elsevier Publishing Comp., 1973.

[10] J. Carrillo. Entropy solutions for nonlinear degenerate problems. Arch. Rational Mech. Anal., 147(4): 269-361, 1999

[11] G. Q. Chen, Q. Ding and K. H. Karlsen. On nonlinear stochastic balance laws. Arch. Rational Mech. Anal. 204 (2012), no. 3, 707-743.

[12] G. Q. Chen and K. H. Karlsen. Quasilinear anisotropic degenerate parabolic equations with time-space dependent diffusion coefficients. Commun. Pure Appl. Anal. 4 (2005), no. 2, 241-266.

[13] G. -Q. Chen and B. Perthame. Wellposedness for non-isotropic degenerate parabolic-hyperbolic equations. Ann. Inst. H. Poincaré Anal. Non Linéaire, 20(4): 645-668, 2003.

[14] A. Debussche, J. Hofmanová and J. Vovelle. Degenerate parabolic stochastic partial differential equations: Quasilinear case . arXiv:1309.5817V1.

[15] A. Debussche and J. Vovelle. Scalar conservation laws with stochastic forcing. J. Funct. Analysis, 259 (2010), 1014-1042.

[16] Z. Dong and T. G. Xu. One-dimensional stochastic Burgers equation driven by Lévy processes. J. Funct. Anal., 243(2):631-678, 2007.

[17] D. Fellah and É. Pardoux. Une formule d'Itô dans des espaces de Banach, et application Stochastic analysis and related topics (Silivri, 1990), Progr. Probable. 255(2):313-373, 2008. 
[18] J. Feng and D. Nualart. Stochastic scalar conservation laws. J. Funct. Anal., 255(2):313-373, 2008.

[19] H. Holden and N. H. Risebro. Conservation laws with random source. Appl. Math. Optim, 36(1997), $229-241$.

[20] W-E. K. Khanin, A. Mazel, and Y. Sinai Invariant measures for Burgers equation with stochastic forcing. Ann. of Math. (2), 151(3): 877-960, 2000.

[21] J. U. Kim. On a stochastic scalar conservation law, Indiana Univ. Math. J. 52 (1) (2003) $227-256$.

[22] S. Peszat and J. Zabczyk. Stochastic partial differential equations with Lévy noise, volume 113 of Encyclopedia of Mathematics and its Applications. Cambridge University Press, Cambridge, 2007. An evolution equation approach.

[23] G. Vallet and P. Wittbold. On a stochastic first-order hyperbolic equation in a bounded domain. Infinite Dimensional Analysis, Quantum Probability, 12(4):1-39, 2009.

[24] G. Vallet. Stochastic perturbation of nonlinear degenerate parabolic problems. Differential Integral Equations 21 (2008), no. 11-12, 1055-1082.

[25] G. Vallet. Dirichlet problem for a degenerated hyperbolic-parabolic equation. Adv. Math. Sci. Appl., 15(2):423450,2005

(Imran H. Biswas)

Centre for Applicable Mathematics, Tata Institute of Fundamental Research, P.O. Box 6503, GKVK Post Office, Bangalore 560065, India

E-mail address: imran@math.tifrbng.res.in

(Ananta K. Majee)

Mathematisches Institut Universität Tübingen Auf Der Morgenstelle 10 D-72076 Tübingen, Germany

E-mail address: majee@na.uni-tuebingen.de

(Guy Vallet)

LMAP UMR- CNRS 5142, IPRA BP 1155, 64013 PAU Cedex, France

E-mail address: guy.vallet@univ-pau.fr 